\title{
Memetic search for the max-bisection problem
}

\author{
Qinghua Wu and Jin-Kao Hao* \\ LERIA, Université d'Angers \\ 2 Boulevard Lavoisier, 49045 Angers Cedex 01, France \\ Computers and Operations Research 40(1): 166-179, 2013 \\ DOI: http://dx.doi.org/10.1016/j.cor.2012.06.001
}

\begin{abstract}
Given an undirected graph $G=(V, E)$ with weights on the edges, the maxbisection problem (MBP) is to find a partition of the vertex set $V$ into two subsets $V_{1}$ and $V_{2}$ of equal cardinality such that the sum of the weights of the edges crossing $V_{1}$ and $V_{2}$ is maximized. Relaxing the equal cardinality constraint leads to the maxcut problem (MCP). In this work, we present a memetic algorithm for MBP which integrates a grouping crossover operator and a tabu search optimization procedure. The proposed crossover operator preserves the largest common vertex groupings with respect to the parent solutions while controlling the distance between the offspring solution and its parents. Extensive experimental studies on 71 well-known G-set benchmark instances demonstrate that our memetic algorithm improves, in many cases, the current best known solutions for both MBP and MCP.

Keywords: Max-bisection, max-cut, memetic search, evolutionary computing, tabu search, optimization.
\end{abstract}

\section{Introduction}

Let $G=(V, E)$ be an undirected graph with vertex set $V=\{1, \ldots, n\}$ and edge set $E \subset V \times V$, each edge $\{i, j\} \in E$ being associated with a weight $w_{i j} \in Z$. The well-known max-cut problem (MCP) is to partition the vertex set $V$ into two disjoint subsets $V_{1} \subset V$ and $V_{2}=V \backslash V_{1}$ such that the sum of the weights

* Corresponding author.

Email addresses: wu@info.univ-angers.fr (Qinghua Wu), hao@info.univ-angers.fr (Jin-Kao Hao). 
of the edges from $E$ that have one endpoint in each subset is maximized, i.e., $\max \sum_{i \in V_{1}, j \in V_{2}} w_{i j}$. MCP is one of the first $21 \mathrm{NP}$-complete problems studied in [21]. When the two subsets $V_{1}$ and $V_{2}$ are required to have the same cardinality (assuming that $n$ is even), the max-cut problem becomes the max-bisection problem (MBP) which remains NP-complete in the general case [13]. Both the max-bisection and max-cut problems has many applications such as statistical physics, classification, social network analysis, and VLSI design [6].

In this work, we are basically interested in the max-bisection problem. Given max-cut is a relaxed max-bisection problem, advances in solving max-bisection can benefit directly the solving of the max-cut problem.

There are two related 'dual' partition problems known as minimum cut and minimum bisection that aim to determine a two-way partition of a graph while minimizing the sum of the weights of the cutting edges (equal cardinality constraint is required for minimum bisection). Notice that in the general case, these minimization problems are different from the max-bisection and maxcut problems considered in this work which concern the two-way partition problems with the maximization criterion. Finally, graph partition problems with the minimization criterion have received much attention in the literature, leading to several well-known public-domain software packages like Chaco [18], Jostle [37], and Metis [22] (see [4] for a recent review).

The computational challenge of the general max-cut and max-bisection problems has motivated a variety of solution approaches including exact methods, approximation algorithms and metaheuristic methods. Examples of approximation algorithms based on semidefinite programming are described in $[10,15,16,20,39]$. These approaches provide a performance guarantee, but do not compete well with other methods in computational testing. Two recent examples of exact methods are described in [24,34] which are based on the cut and price approach and the branch and bound approach respectively. While these methods have the theoretical advantage of finding optimal solutions to a given problem, their applications are generally limited to problems with no more than a few hundred vertices.

For larger problem instances, a number of heuristics and metaheuristics are often used to find approximate solutions of good quality with a reasonable computing time. This includes for the max-bisection problem deterministic annealing [7], Lagrangian net [38] and variable neighborhood search [25]. There are many heuristics and metaheuristics for the max-cut problem including simulated annealing [1], rank-2 relaxation heuristic [5], GRASP [9], diversification driven tabu search [23], advanced scatter search [28], global equilibrium search [35], and probabilistic tabu search [36].

In this paper, we present a memetic algorithm for the max-bisection problem 
(denoted by MAMBP). The proposed algorithm integrates three complementary key components which jointly ensure the high efficiency of the search process. First, to generate promising new solutions, we introduce a dedicated crossover operator which tries to preserve groups of vertices that are shared by parent solutions. The design of this crossover operator relies on the observation that, given a set of high quality bisections of a graph, there is always a large number of vertices grouped together throughout these bisections. Second, we devise a tabu search optimization procedure for the purpose of intensified search around a given solution. The tabu search procedure uses a vertex move neighborhood and incremental evaluation techniques for a fast neighborhood examination. Finally, to maintain a healthy diversity of the population, we employ a pool updating strategy which takes into account both the solution quality and the distance between solutions.

We show extensive experimental results on 71 well-known G-set benchmark graphs (with 800 to 20000 vertices) in the literature, showing that the proposed algorithm achieves highly competitive results with respect to the existing max-bisection heuristics. Moreover, when considering the relaxed max-cut problem, the results produced by our MAMBP algorithm remain highly competitive even when they are compared to those obtained by dedicated maxcut algorithms; for 31 max-cut instances, MAMBP improves the previous best known max-cut solutions of the literature.

In the next section, the components of our memetic algorithm are described, including the tabu search procedure, the crossover operator and the pool replacement strategy. Section 3 is dedicated to computational results and detailed comparisons with other state-of-the-art algorithms in the literature. Section 4 investigates several essential parts of the proposed memetic algorithm, followed by concluding remarks given in Section 5 .

\section{Memetic Algorithm}

Memetic algorithms are known to be an effective approach in solving a number of hard combinatorial optimization problems [29,30,17]. Typically, a memetic approach repeatedly alternates between a recombination (or crossover) operator to generate solutions located in promising regions in the search space and a local optimization procedure to search around the newly generated solutions. It is commonly admitted that the success of this approach depends critically on the recombination operator. In order to be effective, the recombination operator must be adapted to the problem being solved and should be able to transmit meaningful features from parents to offspring.

The general scheme of our memetic approach for MBP is summarized in Algo- 
rithm 1. Basically, our memetic algorithm begins with an initial population of solutions which are first improved by the local optimization procedure based on tabu search [14] (lines 1-5, Sections 2.2 and 2.3) and then repeats an iterative process for a fixed number of times (generations) (lines 6-13). At each generation, two solutions are selected to serve as parents (Section 2.4). The crossover operator is applied to the parents to generate a new offspring solution (Section 2.5) which is further improved by the tabu search optimization procedure (Section 2.3). Finally, we apply a quality-and-diversity based rule to decide whether the improved offspring solution can be inserted into the population (Section 2.6). In the following subsections, we give more details on the components of our memetic algorithm.

\subsection{Search space and cost function}

Recall that MBP consists in partitioning the vertex set $V$ into two subsets of equal cardinality such that the weights on the edges between the two subsets is maximized. As such, we define the search space explored by our memetic algorithm as the set of all possible partitions of $V$ into 2 disjoint subsets of equal cardinality (also called bisections), i.e., $\Omega=\left\{\left\{V_{1}, V_{2}\right\}:\left|V_{1}\right|=\left|V_{2}\right|=\right.$ $\left.\frac{|V|}{2}, V_{1} \cap V_{2}=\varnothing\right\}$. Clearly, the size of $\Omega$ is given by $C(|V|,|V| / 2)$.

Given a bisection $I=\left\{V_{1}, V_{2}\right\} \in \Omega$, the cost function (also called the fitness function) $f(I)$ sums up the weights of the edges between the two subsets $V_{1}$ and $V_{2}$ such that:

$$
f(I)=\sum_{i \in V_{1}, j \in V_{2}} w_{i j}
$$

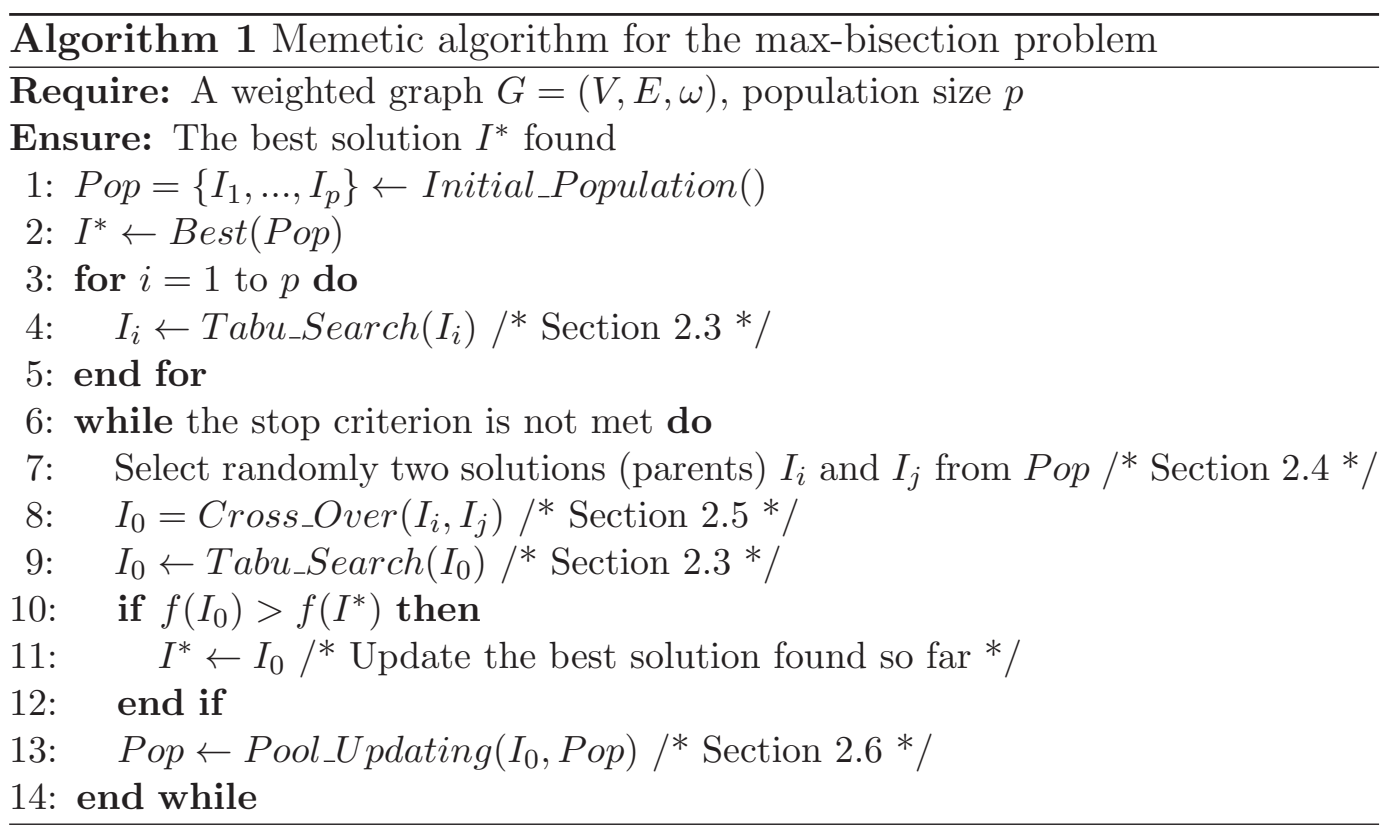


Then, for two bisections $I_{A} \in \Omega$ and $I_{B} \in \Omega, I_{A}$ is better than $I_{B}$ if and only if $f\left(I_{A}\right)>f\left(I_{B}\right)$. The goal of the max-bisection problem is to find:

$$
\underset{I \in \Omega}{\arg \max } f(I)
$$

Given the size of the search space $\Omega$, it is particularly challenging to find an exact solution or an approximate solution of high quality.

\subsection{Initial population}

The solutions (individuals) of the initial population are created as follows. For each individual, an equal sized partition is first created at random and then improved by the tabu search procedure (see Section 2.3). The improved solution is added into the population if this solution is not already present in it. Otherwise, this solution is discarded and a new random (equal sized) partition is created. This procedure is iterated until the population is filled with $p$ solutions ( $p$ is the population size). This simple procedure provides an initial population of diverse solutions of good quality.

\subsection{The Perturbation-based Tabu Search Procedure}

Our tabu search (TS) procedure aims to improve a given solution $I$ and plays the key role of local optimization within our memetic algorithm. Basically, our tabu search procedure repeatedly alternates between an intensification phase ensured by the basic tabu search and a diversification phase controlled by a perturbation mechanism [14]. Algorithm 2 describes this perturbationbased tabu search procedure, whose components are detailed in the following subsections.

\subsubsection{Neighborhood and move}

Given a bisection $I=\left\{V_{1}, V_{2}\right\}$ with $\left|V_{1}\right|=\left|V_{2}\right|$, the basic idea of the neighborhood explored by our tabu search consists in moving first a vertex from subset $V_{1}$ to $V_{2}$, accompanied by moving another vertex from $V_{2}$ to $V_{1}$. Note that in such a way, the balance between the two parts of the bisection is always maintained (i.e., $\left.\left|V_{1}\right|=\left|V_{2}\right|\right)$.

The key concept related to our neighborhood is the move gain, which indicates how much a solution (bisection) is improved according to the optimization objective $f$ (Eq. 1, Section 2.1) if a vertex is moved from its subset to the 
other subset. For large problem instances, it is imperative to be able to rapidly determine the move gain of a move. In our implementation, we use a fast incremental evaluation technique which is based on a streamlined calculation for updating the move gain after each move. More formally, let $\Delta_{v}$ be the move gain of moving vertex $v$ to the other subset. Then initially, each move value can be calculated in linear time using the formula:

$$
\Delta_{v}=\left\{\begin{array}{cl}
\sum_{x \in V_{1}, x \neq v} w_{v x}-\sum_{y \in V_{2}} w_{v y}, & \text { if } v \in V_{1} \\
\sum_{y \in V_{2}, y \neq v} w_{v y}-\sum_{x \in V_{1}} w_{v x}, & \text { otherwise. }
\end{array}\right.
$$

Once a move is performed, one just needs to update a subset of move gains

$\overline{\text { Algorithm } 2 \text { Perturbation-based tabu search for the max-bisection problem }}$ Require: A weighted graph $G=(V, E, \omega)$, initial bisection $I=\left\{V_{1}, V_{2}\right\}$, number $l r$ of tabu search iterations, number $c r$ of consecutive iterations before triggering a perturbation

Ensure: The best bisection $I^{*}$

1: Iter $\leftarrow 0 \quad / *$ Iteration counter */

2: $I^{*} \leftarrow I \quad /^{*} I^{*}$ records the best bisection found so far */

3: Initiate the tabu list and tabu tenure $/ *$ See Section 2.3.2*/

4: for each vertex $v \in V$ do

5: $\quad$ Compute the move gain $\Delta_{v}$ according to Eq. 2.

6: end for

7: while Iter $<\operatorname{lr}$ do

8: Choose a best allowed (i.e., not forbidden by the tabu list) vertex $v_{1} \in V_{1}$ according to max-move gain criterion (ties are broken randomly) $/ *$ See this section */

9: Generate intermediate solution $I$ by moving $v_{1}$ from $V_{1}$ to $V_{2}$ (i.e., $V_{1}=$ $V_{1} \backslash\left\{v_{1}\right\}$ and $\left.V_{2}=V_{2} \cup\left\{v_{1}\right\}\right)$

10: Add $v_{1}$ in the tabu list and update the move gain $\Delta_{v}$ for each $v \in V$

11: Choose a best allowed vertex $v_{2} \in V_{2}$ (i.e., $v_{2}$ is not forbidden by the tabu list or $v_{2}$ leads to a new solution better than solution $I^{*}$ ) according to max-move gain criterion (ties are broken randomly)

12: Generate new solution $I$ by moving $v_{2}$ from $V_{2}$ to $V_{1}$, (i.e., $V_{1}=V_{1} \cup\left\{v_{2}\right\}$ and $\left.V_{2}=V_{2} \backslash\left\{v_{2}\right\}\right)$

13: Add $v_{2}$ in the tabu list and update the move gain $\Delta_{v}$ for each $v \in V$

14: $\quad$ if $f(I)>f\left(I^{*}\right)$ then

15: $\quad I^{*} \leftarrow I \quad / *$ Update the best solution found so far */

16: end if

17: $\quad$ Iter $\leftarrow$ Iter +1

18: $\quad$ if $I^{*}$ not improved after $\mathrm{cr}$ iterations then

19: $\quad I \leftarrow \operatorname{Perturb}(I) / *$ Apply perturbations to $I$, Section 2.3.3*/

20: $\quad$ end if

21: end while 
affected by this move. Specifically, the following abbreviated calculation can be performed to update the move gains upon moving $v$ from its set to the other set:

- $\Delta_{v}=-\Delta_{v}$

- for each $u \in V-\{v\}$,

$$
\Delta_{u}= \begin{cases}\Delta_{u}-2 \times w_{u v}, & \text { if } u \text { is in the same set as } v \text { before moving } v \\ \Delta_{u}+2 \times w_{u v}, & \text { otherwise. }\end{cases}
$$

An illustration of the proposed approach for initializing and updating the move gains is provided in Figure 1.
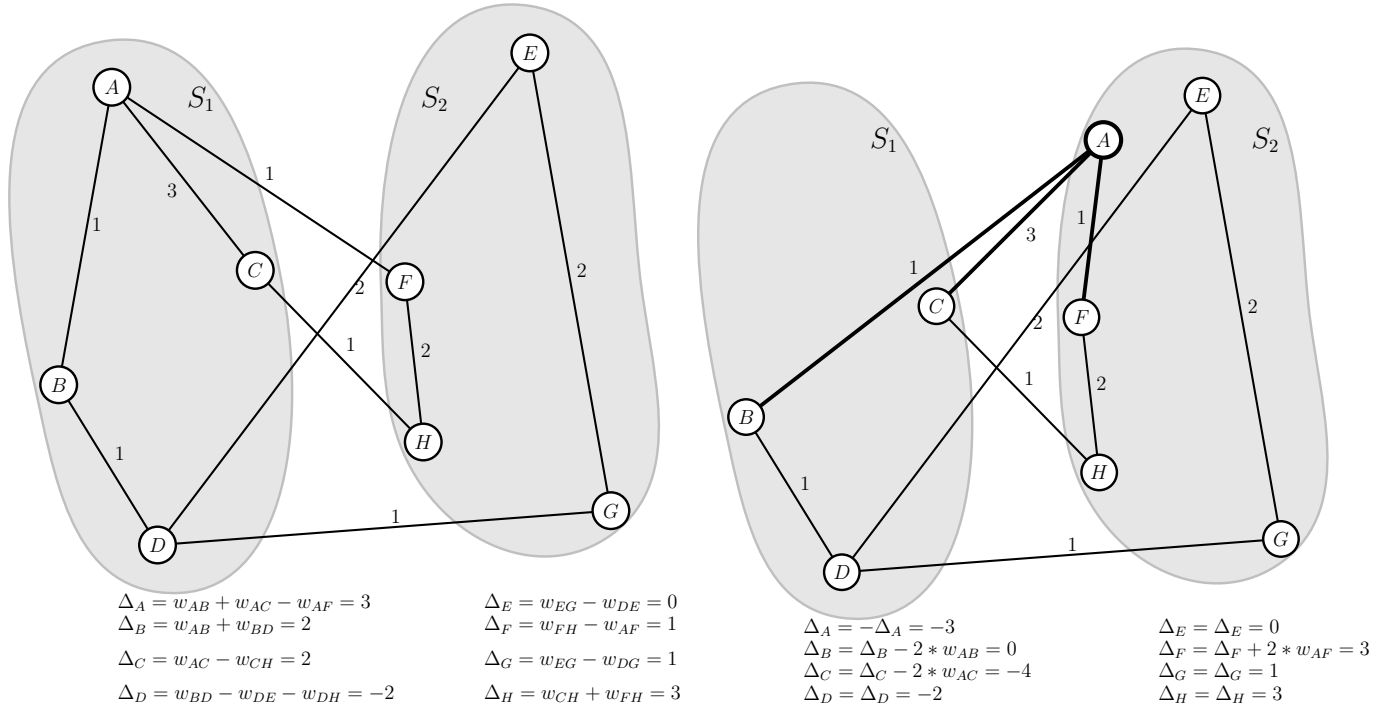

Fig. 1. An example of the initialization (left) and update (right) of the move gain

Our tabu search procedure then restricts consideration to those vertices which are not forbidden by the tabu list (see Section 2.3.2) and have the largest $\Delta$ value. At each iteration of our tabu search, we first select a non tabu vertex in $V_{1}$ with the largest $\Delta$ value and move it from $V_{1}$ to $V_{2}$. Then, we choose another non tabu vertex in $V_{2}$ with the best move gain and move it from $V_{2}$ to $V_{1}$. In the case that two or more non tabu moves have the same largest $\Delta$ value, one of them is chosen at random. Once a vertex $v$ is changed from its original set to the other set, it is classified tabu for the next $t l$ iterations, during which $v$ is forbidden to be moved back to its original set. A simple aspiration criterion is applied that permits a vertex to be selected in spite of being tabu if it leads to a solution better than the current best solution. Notice that aspiration is only applicable to the selection of the second vertex from $V_{2}$ (see Algorithm 2, line 11) since the selection of the first vertex from $V_{1}$ leads to an intermediate imbalanced bisection which is not a feasible solution. The TS process stops when a maximum allowed number $(l r)$ of iterations is reached. 


\subsubsection{Tabu list and tabu tenure management}

Within TS, a tabu list is introduced to forbid the previously visited solutions to be revisited. In our TS algorithm, each time a vertex $v$ goes from its original subset to the opposite set, it is forbidden to bring back $v$ to its original set for a certain number of iterations (called the tabu tenure). Inspired by the tabu mechanism proposed in [11], we employ a particular technique whose goal is to vary the tabu tenure throughout the search.

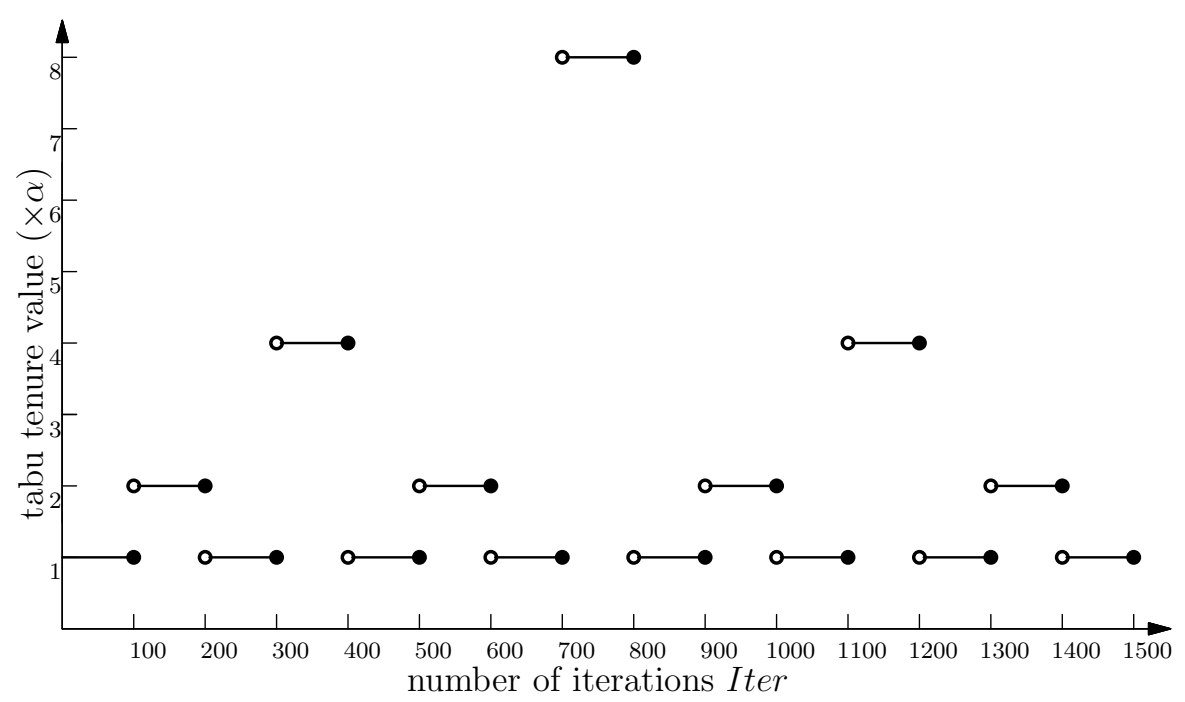

Fig. 2. An illustration of the step function (one period) used for tuning the tabu tenure.

The tabu tenure is dynamically tuned by a periodic step function $T$ defined over the number of iterations Iter (see Figure 2 for an example). Each period of the step function is composed of 1500 iterations divided into 15 steps or intervals $\left[x_{i} . . x_{i+1}-1\right]_{i=1,2, \ldots, 15}$ with $x_{1}=1, x_{i+1}=x_{i}+100$. The tabu tenure changes dynamically during the search and according to the current iteration number, it takes one of four possible values $(\alpha, 2 \times \alpha, 4 \times \alpha, 8 \times \alpha)$ where $\alpha$ is a parameter. Precisely, for an iteration Iter $\in\left[x_{i} . . x_{i+1}-1\right]$, the tabu tenure T(Iter) is given by $\left(y_{i}\right)_{i=1,2, \ldots, 15}=\alpha \times(1,2,1,4,1,2,1,8,1,2,1,4,1,2,1)$. Therefore, the tabu tenure is equal to $\alpha$ for the first 100 iterations [1..100], then $2 \times \alpha$ for iterations from [101..200], followed by $\alpha$ again for iterations [201..300] and $4 \times \alpha$ for iterations [401..500] etc. After reaching the largest value $8 \times \alpha$ for iterations [701..800], the tabu tenure drops again to $\alpha$ for the next 100 iterations and so on. This function repeats periodically this variation scheme after every 1500 iterations.

We have also experimented the four values $(\alpha, 2 \times \alpha, 4 \times \alpha, 8 \times \alpha)$ in a static way, i.e., applying each of them during the whole search. Experimental results showed that the dynamic tabu tenure method performs better than the static method and allows the search to effectively escape from local optima. 


\subsubsection{Perturbation mechanism}

The above basic tabu search is generally able to attain solutions of good quality. However, it may happen that it gets stuck in deep local optima. In our case, the search is judged stagnating each time the best bisection $I^{*}$ found so far is not further improved after a number $\mathrm{cr}$ of consecutive iterations.

To help the search to escape from such deep local optima, we apply a simple perturbation mechanism to the current solution to bring diversification into the search. The perturbation consists in swapping a number of pairs of vertices in the following way. For each swap, we randomly choose one vertex $u$ from $V_{1}$ and another vertex $v$ from $V_{2}$, and then swap $u$ and $v$ (i.e., $V_{1}=V_{1} \bigcup\{v\} \backslash\{u\}$ and $V_{2}=V_{2} \bigcup\{u\} \backslash\{v\}$ ). This process is repeated $\gamma$ times where $\gamma$ is a parameter which indicates the strength of the perturbation.

\subsection{Parent selection}

Our memetic algorithm is a steady-state algorithm such that at each generation one new solution is generated by combining two parent solutions chosen from the population. To choose the parents, several selection methods can be applied. In our case, we have experimented three well-known strategies: roulette-wheel, tournament and random [2]. With roulette-wheel selection, an individual is selected with a probability which is proportional to its fitness (objective) value. For tournament selection (the standard 2-way deterministic tournament), two individuals are taken at random and the best of the two is selected. Random selection chooses each individual with equal probability. Experimental results with these selection methods have shown that within the context of this work, no significant difference was observed. This can be explained intuitively as follows. In fact, since the individuals are improved by the powerful tabu search procedure, they are generally of good quality, implying that their fitnesses are not so different in the objective space (though the bi-sections they represent may be quite different due to the pool updating strategy used in Section 2.6). Under such a circumstance, it is clear that roulette-wheel, tournament and random would lead to a similar selection effect. For this reason, we have decided to retain the simple random selection in our algorithm.

\subsection{Crossover operator}

Crossover is another key component of our memetic algorithm. In practice, instead of applying blind crossovers, it is often preferable to devise a dedicated recombination operator that have strong "semantics" with respect to the prob- 
lem being solved [17]. In the case of MBP, a bisection of a graph $G=(V, E)$ corresponds to a partition of $V$ into two disjoint groups of vertices. With this point of view, MBP can be considered as a grouping problem [8]. For grouping problems, the crossover operators should manipulate groups of objects rather than individual objects. Such an approach for designing crossover operators has been successfully applied to solve a number of grouping problems such as graph coloring $[12,27,32]$, bin packing [8] and graph partitioning $[3,11]$.

In this work, we propose a dedicated grouping crossover operator for MBP, the proposed crossover operator is constrained to conserve subsets (or grouping vertices) of the vertex partitions of parents in offspring solutions. The rationale behind this approach is based on a careful analysis of local optimal solutions which discloses that high quality local optima share many grouping vertices (see Section 4). Therefore, if a set of vertices are always grouped together throughout a set of high quality solutions, these vertices are considered to have a high chance to be part of a globally optimal solution.

The proposed crossover operator follows the general principle presented above and operates in two sequential steps. We first create a partial bisection of maximal size by conserving the largest common vertex groupings with respect to the two selected parents. In order to obtain a full bisection, we complete this partial bisection by redistributing the remaining unassigned vertices based on a greedy construction strategy.

More precisely, given two parents $I^{a}=\left\{V_{1}^{a}, V_{2}^{a}\right\}$ and $I^{b}=\left\{V_{1}^{b}, V_{2}^{b}\right\}$, the partial offspring $I^{c}=\left\{V_{1}^{c}, V_{2}^{c}\right\}$ is constructed such that $V_{1}^{c}=V_{1}^{a} \cap V_{1}^{b}$ and $V_{2}^{c}=$ $V_{2}^{a} \cap V_{2}^{b}$. It should be noted that if $\left|V_{1}^{a} \cap V_{1}^{b}\right|<\left|V_{2}^{a} \cap V_{2}^{b}\right|$, we first exchange the two parts of $I^{b}$ before constructing the offspring $I^{c}$ for the purpose of preserving the largest common grouping vertices (see Figure 2 for an example). For the constructed partial offspring $I^{c}=\left\{V_{1}^{c}, V_{2}^{c}\right\}$, it can be shown that $\left|V_{1}^{c}\right|=\left|V_{2}^{c}\right|$.

When the partial offspring $I^{c}=\left\{V_{1}^{c}, V_{2}^{c}\right\}$ is constructed, the vertices in $V_{1}^{a} \backslash$ $V_{1}^{c}$ and $V_{2}^{a} \backslash V_{2}^{c}$ are left unassigned. For the vertices in $V_{1}^{a} \backslash V_{1}^{c}$, they are grouped together in both parent solutions $I^{a}$ and $I^{b}$ (in fact, $V_{1}^{a} \backslash V_{1}^{c} \subset V_{1}^{a}$ and $V_{1}^{a} \backslash V_{1}^{c} \subset V_{2}^{b}$, see Figure 3 for an example). Similarly, the vertices in $V_{2}^{a} \backslash V_{2}^{c}$ are also grouped together in both $I^{a}$ and $I^{b}$. Thus, these left vertices should be dealt with care in order to generate a diversified offspring solution. In the following, we use a diversification-guided strategy to redistribute these unassigned vertices by taking into account both the solution quality of the offspring and its distance to its parent solutions.

Given the partial solution $I^{c}=\left\{V_{1}^{c}, V_{2}^{c}\right\}$, we use two greedy functions that take into account the contribution of the left vertices to the objective function of $I^{c}$. Specifically, for each $v \in V_{1}^{a} \backslash V_{1}^{c}$, we define $\sigma(v)=\sum_{u \in V_{1}^{c}} w_{u v}$ and $\sigma^{\prime}(v)=$ 
$\sum_{u \in V_{2}^{c}} w_{u v}$. Then the vertices in $V_{1}^{a} \backslash V_{1}^{c}$ are assigned to the two parts of $I^{c}$ one by one. At the first step, we examine all the vertices in $V_{1}^{a} \backslash V_{1}^{c}$ to identify the vertex with the largest $\sigma$ value and move it from $V_{1}^{a} \backslash V_{1}^{c}$ to $V_{1}^{c}$. Afterward, $V_{2}^{c}$ is considered, we identify the vertex in $V_{1}^{a} \backslash V_{1}^{c}$ with the largest $\sigma^{\prime}$ value and assign it to $V_{2}^{c}$. Then at each step of our procedure, $V_{1}^{c}$ and $V_{2}^{c}$ are considered in turn to distribute the remaining vertices in $V_{1}^{a} \backslash V_{1}^{c}$ (see Figure 2). Once all vertices in $V_{1}^{a} \backslash V_{1}^{c}$ are assigned to the two subsets of $I^{c}$, the same procedure is applied to handle the vertices in $V_{2}^{a} \backslash V_{2}^{c}$. Finally, one observes that the offspring $I^{c}$ generated by our crossover operator has essentially the same distance to its two parents and is of relatively high quality.

\subsection{Pool updating strategy}

When an offspring solution is created by the crossover operator presented in the last section and improved by the tabu search algorithm described in Section 2.3, we decide whether the improved offspring should be inserted into the population and which existing solution of the population should be replaced. Basically, our decisions are made based on both solution quality and distance between solutions in the population $[26,27,32]$. Therefore, we first define the distance between two solutions before presenting the used pool updating strategy.

Definition 1. Distance between two bisections: Given two bisections $I^{a}=\left\{V_{1}^{a}, V_{2}^{a}\right\}$ and $I^{b}=\left\{V_{1}^{b}, V_{2}^{b}\right\}$, the distance between $I^{a}$ and $I^{b}$ (call it $d$ ) can

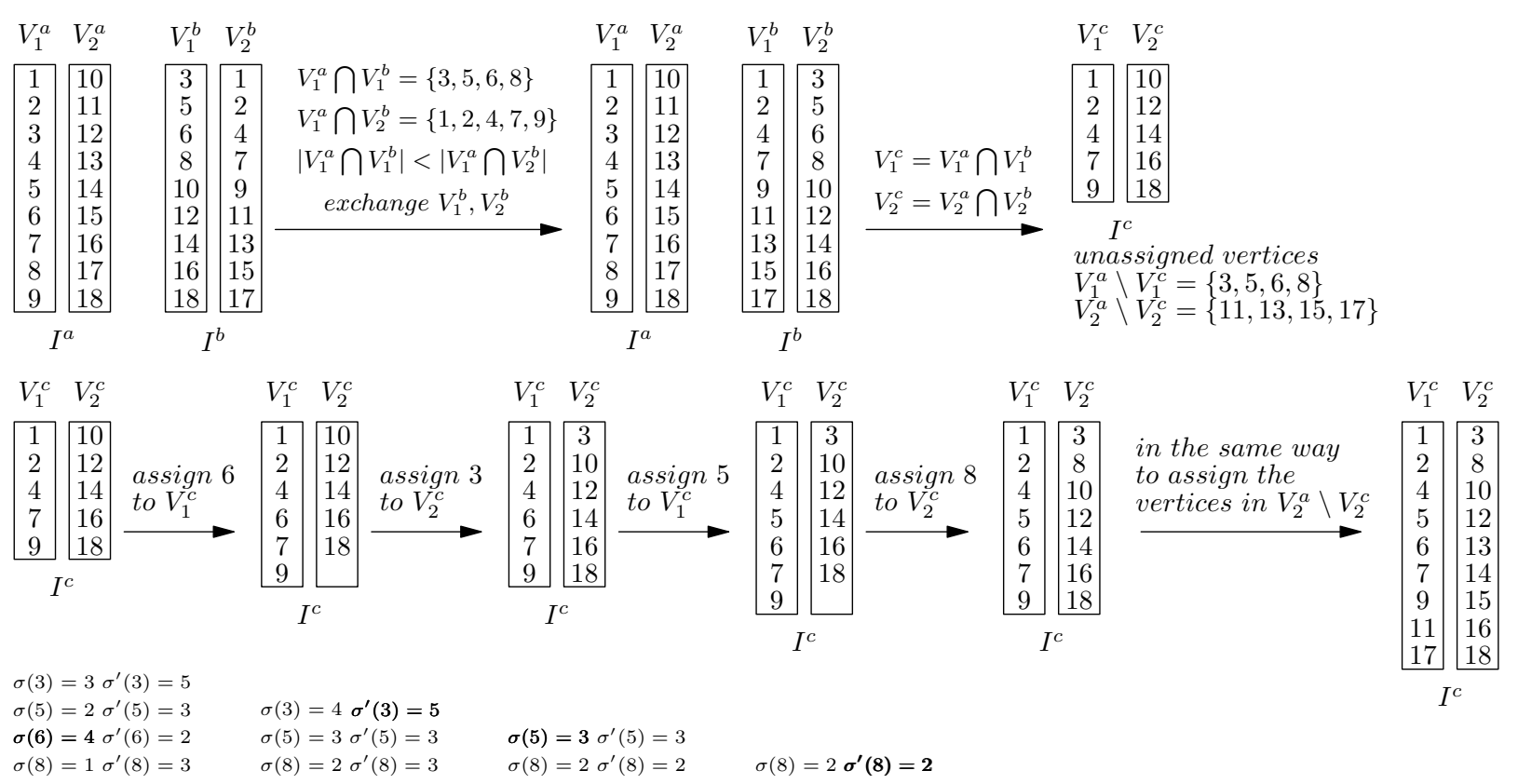

Fig. 3. An illustration of the crossover operator. 
be defined as the minimum number of one-move steps necessary to transform $I^{b}$ to $I^{a}$. A move step corresponds to the operation of moving one vertex from one side of the bisection to the other side. More formally, the distance $d\left(I^{a}, I^{b}\right)$ is determined using the formula $d\left(I^{a}, I^{b}\right)=|V|-s\left(I^{a}, I^{b}\right)$ where $s\left(I^{a}, I^{b}\right)$ is a similarity function which corresponds to the number of vertices that do not need to be moved to transform $I^{b}$ to $I^{a}$ [33]. This similarity function can be defined as follows. If $\left|V_{1}^{a} \cap V_{1}^{b}\right|<\left|V_{1}^{a} \cap V_{2}^{b}\right|$, we first exchange the two parts of $I_{b}$, then $s\left(I^{a}, I^{b}\right)$ is defined as $s\left(I^{a}, I^{b}\right)=\left|\left(V_{1}^{a} \cap V_{1}^{b}\right) \cup\left(V_{2}^{a} \cap V_{2}^{b}\right)\right|$.

Definition 2. Distance between one bisection and a population: Given a population $P_{o p}=\left\{I^{1}, \ldots, I^{p}\right\}$ and the distance $d_{i j}$ between any two bisections $I^{i}$ and $I^{j}(i, j=1, \ldots, p, i \neq j)$, the distance between a bisection $I^{i}$ $(i=1, \ldots, p)$ and the population Pop is defined as the minimum distance between $I^{i}$ and any other bisection in the population:

$$
D_{i, P o p}=\min \left\{d_{i j} \mid I^{j} \in P o p, j \neq i\right\}
$$

The general scheme of our pool updating strategy is described in Algorithm 3. In order to update the population, the new generated offspring $I^{0}$ is tentatively inserted into the population, i.e., $P o p^{\prime}=P o p \bigcup\left\{I^{0}\right\}$. Then, the worst solution (denoted by $I^{w}$ ) in the population $P o p^{\prime}=\left\{I^{0}, \ldots, I^{p}\right\}$ is identified. If $I^{w}$ is not the offspring $I^{0}$, then $I^{0}$ is inserted into the population and replaces the worst solution $I^{w}$, i.e., Pop $=$ Pop $\cup\left\{I^{0}\right\} \backslash\left\{I^{w}\right\}$. To determine the worst solution in the population, we use a quality-and-distance scoring function which was originally proposed in [26] and is defined as follows:

$$
g\left(I^{i}\right)=\beta \widetilde{A}\left(f\left(I^{i}\right)\right)+(1-\beta) \widetilde{A}\left(D_{i, P o p}\right)
$$

where $f\left(I^{i}\right)$ is the objective function value defined in section $2.1, \beta$ is a param-

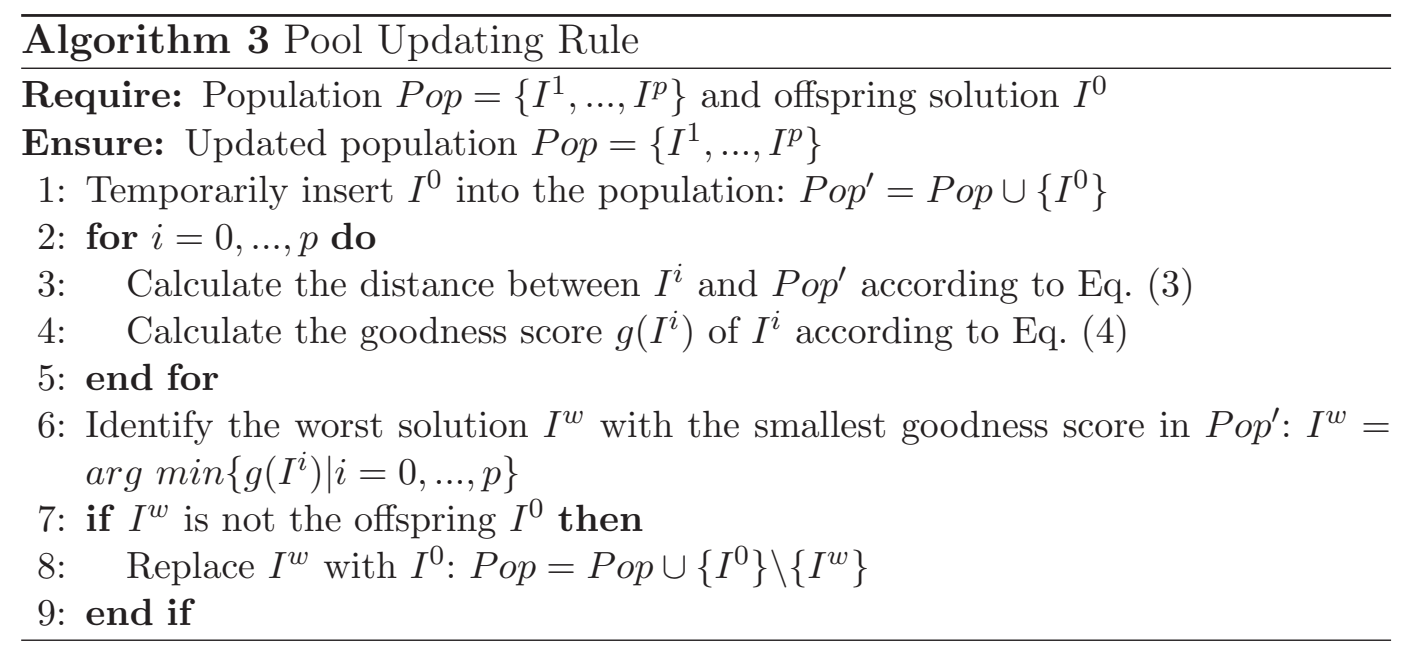


Table 1

Settings of important parameters

\begin{tabular}{|c|c|c|c|}
\hline Parameters & Section & Description & Values \\
\hline$p$ & 2.2 & size of population & 10 \\
\hline$\alpha$ & 2.3 & tabu tenure management factor & 15 \\
\hline$c r$ & 2.3 & $\begin{array}{c}\text { non-improvement TS iterations before } \\
\text { perturbation }\end{array}$ & 3,000 \\
\hline$\gamma$ & 2.3 & perturbation strength & 200 \\
\hline$l r$ & 2.3 & number of TS iterations after recombination & $10^{6}$ \\
\hline$\beta$ & 2.6 & goodness score coefficient & $0.6[26]$ \\
\hline
\end{tabular}

eter set to 0.6 according to [26], and $\widetilde{A}($.$) represents the normalized function:$

$$
\widetilde{A}(y)=\frac{y-y_{\min }}{y_{\max }-y_{\min }+1}
$$

where $y_{\min }$ and $y_{\max }$ are respectively the minimum and maximum of $y$ in the population Pop. " +1 " is used to avoid the possibility of a 0 denominator.

\section{Experimental results}

\subsection{Test Instances}

To evaluate the efficiency of the proposed memetic approach, extensive experiments were carried out on 71 well-known G-set benchmark graphs that are frequently used to assess max-bisection and max-cut algorithms. The $G$-set contains graphs ranging in size from 800 vertices to 20000 vertices $^{1}$. These instances are generated by a machine-independent graph generator, comprising of toroidal, planar and random weighted graphs. Many authors including $[38,5,9,23,28,31,35,36]$ employ these instances to test their algorithms for the max-bisection problem as well as the max-cut problem.

\subsection{Parameter settings}

The parameter settings used in our experiments are given in Table 1. These parameter values have been determined by performing a preliminary experiment on a selection of 11 (hard) instances $\left(G_{32}, G_{33}, G_{35}, G_{40}, G_{41}, G_{42}\right.$, $G_{55}, G_{57}, G_{62}, G_{65}$ and $\left.G_{66}\right)$. To determine the parameter values, we first began with the parameters required by the TS algorithm $(\alpha, c r, \gamma$, see Section 2.3) and then determined the parameters used by the memetic algorithm $(p, l r, \beta)$. For the TS algorithm we tested values for $\alpha$ in the range [10..30], $c r$ in the range [2000..6000] and $\gamma$ in the range [100..300]. For each value of

$\overline{1}$ Available at http://www.stanford.edu/ yyye/yyye/Gset/ 
Table 2

Parameter tuning: $\alpha$

\begin{tabular}{|c|c|c|c|c|c|c|c|c|c|c|}
\hline \multirow[t]{3}{*}{ Instance } & \multicolumn{10}{|c|}{ TS with different $\alpha$ values } \\
\hline & \multicolumn{2}{|c|}{$\alpha=10$} & \multicolumn{2}{|c|}{$\alpha=15$} & \multicolumn{2}{|c|}{$\alpha=20$} & \multicolumn{2}{|c|}{$\alpha=25$} & \multicolumn{2}{|c|}{$\alpha=30$} \\
\hline & $f_{\text {best }}$ & $f_{\text {avg }}$ & $f_{\text {best }}$ & $f_{\text {avg }}$ & $f_{\text {best }}$ & $f_{\text {avg }}$ & $f_{\text {best }}$ & $f_{\text {avg }}$ & $f_{\text {best }}$ & $f_{\text {avg }}$ \\
\hline$G_{32}$ & 1396 & 1391.20 & 1400 & 1392.80 & 1400 & 1393.10 & 1400 & 1392.70 & 1398 & 1393.90 \\
\hline$G_{33}$ & 1374 & 1371.20 & 1376 & 1371.80 & 1374 & 1372.40 & 1376 & 1373.60 & 1374 & 1372.30 \\
\hline$G_{35}$ & 7669 & 7659.30 & 7673 & 7663.30 & 7673 & 7664.05 & 7668 & 7664.45 & 7673 & 7666.25 \\
\hline$G_{40}$ & 2383 & 2366.45 & 2388 & 2369.70 & 2392 & 2373.05 & 2389 & 2368.95 & 2386 & 2364.80 \\
\hline$G_{41}$ & 2390 & 2368.55 & 2396 & 2369.20 & 2388 & 2372.25 & 2393 & 2368.60 & 2384 & 2369.70 \\
\hline$G_{42}$ & 2474 & 2459.45 & 2476 & 2465.95 & 2472 & 2463.65 & 2477 & 2465.75 & 2474 & 2465.40 \\
\hline$G_{55}$ & 10257 & 10229.40 & 10263 & 10231.20 & 10265 & 10229.80 & 10263 & 10228.90 & 10259 & 10232.80 \\
\hline$G_{57}$ & 3458 & 3445.90 & 3460 & 3446.20 & 3456 & 3446.00 & 3456 & 3446.50 & 3454 & 3445.60 \\
\hline$G_{62}$ & 4812 & 4794.00 & 4812 & 4792.00 & 4810 & 4791.80 & 4810 & 4796.90 & 4810 & 4795.10 \\
\hline$G_{65}$ & 5504 & 5474.50 & 5504 & 5476.50 & 5498 & 5475.70 & 5498 & 5477.00 & 5492 & 5477.80 \\
\hline$G_{66}$ & 6276 & 6254.00 & 6282 & 6259.50 & 6284 & 6255.00 & 6276 & 6255.90 & 6276 & 6257.40 \\
\hline
\end{tabular}

Table 3

Parameter tuning: $\mathrm{cr}$

\begin{tabular}{|c|c|c|c|c|c|c|c|c|c|c|}
\hline \multirow[t]{3}{*}{ Instance } & \multicolumn{10}{|c|}{ TS with different $c r$ values } \\
\hline & \multicolumn{2}{|c|}{$\mathrm{cr}=2000$} & \multicolumn{2}{|c|}{$c r=3000$} & \multicolumn{2}{|c|}{$c r=4000$} & \multicolumn{2}{|c|}{$c r=5000$} & \multicolumn{2}{|c|}{$c r=6000$} \\
\hline & $f_{\text {best }}$ & $f_{\text {avg }}$ & $f_{\text {best }}$ & $f_{\text {avg }}$ & $f_{\text {best }}$ & $f_{\text {avg }}$ & $f_{\text {best }}$ & $f_{\text {avg }}$ & $f_{\text {best }}$ & $f_{\text {avg }}$ \\
\hline$G_{32}$ & 1396 & 1390.20 & 1400 & 1392.80 & 1400 & 1393.10 & 1400 & 1393.50 & 1402 & 1394.20 \\
\hline$G_{33}$ & 1376 & 1371.40 & 1376 & 1371.80 & 1376 & 1372.70 & 1376 & 1371.90 & 1376 & 1372.20 \\
\hline$G_{35}$ & 7672 & 7663.65 & 7673 & 7663.30 & 7671 & 7663.25 & 7673 & 7662.20 & 7668 & 7660.00 \\
\hline$G_{40}$ & 2394 & 2368.80 & 2388 & 2369.70 & 2388 & 2366.20 & 2385 & 2366.10 & 2385 & 2358.80 \\
\hline$G_{41}$ & 2387 & 2372.10 & 2396 & 2369.20 & 2395 & 2367.90 & 2393 & 2369.95 & 2395 & 2369.00 \\
\hline$G_{42}$ & 2471 & 2463.85 & 2476 & 2465.95 & 2473 & 2456.50 & 2468 & 2456.05 & 2478 & 2456.85 \\
\hline$G_{55}$ & 10265 & 10220.60 & 10263 & 10231.20 & 10263 & 10229.60 & 10261 & 10226.20 & 10257 & 10225.90 \\
\hline$G_{57}$ & 3458 & 3448.00 & 3460 & 3446.20 & 3454 & 3441.90 & 3462 & 3446.00 & 3452 & 3438.40 \\
\hline$G_{62}$ & 4812 & 4798.20 & 4812 & 4792.00 & 4808 & 4792.10 & 4808 & 4792.10 & 4802 & 4788.90 \\
\hline$G_{65}$ & 5500 & 5476.00 & 5504 & 5476.50 & 5494 & 5473.40 & 5492 & 5478.90 & 5484 & 5465.90 \\
\hline$G_{66}$ & 6280 & 6262.00 & 6282 & 6259.50 & 6272 & 6252.10 & 6286 & 6258.80 & 6262 & 6238.70 \\
\hline
\end{tabular}

these parameters, 20 independent runs of TS were performed on each problem instance. Table 2 shows the results for different $\alpha$ values (with $\mathrm{cr}=3000$ and $\gamma=200$ ) while Table 3 indicates the results for different $c r$ values (with $\alpha=15$ and $\gamma=200$ ) and Table 4 shows the results for different $\gamma$ values (with $\alpha=15$ and $c r=3000$ ). The tables show the best and averaged objective values found over the 20 runs. From these tables, we observe that the combination $(\alpha=15, c r=3000, \gamma=200)$ globally leads to the best results. For the parameters related to the memetic algorithm, we fixed $p=10, \operatorname{lr}=10^{6}$ in a similar way while $\beta=0.6$ is chosen according to [26].

These parameter values were used to report our computational results, even 
Table 4

Parameter tuning: $\gamma$

\begin{tabular}{|c|c|c|c|c|c|c|c|c|c|c|}
\hline \multirow[t]{3}{*}{ Instance } & \multicolumn{10}{|c|}{ TS with different $\gamma$ values } \\
\hline & \multicolumn{2}{|c|}{$\gamma=100$} & \multicolumn{2}{|c|}{$\gamma=150$} & \multicolumn{2}{|c|}{$\gamma=200$} & \multicolumn{2}{|c|}{$\gamma=250$} & \multicolumn{2}{|c|}{$\gamma=300$} \\
\hline & $f_{\text {best }}$ & $f_{a v g}$ & $f_{\text {best }}$ & $f_{a v g}$ & $f_{\text {best }}$ & $f_{\text {avg }}$ & $f_{\text {best }}$ & $f_{a v g}$ & $f_{\text {best }}$ & $f_{a v g}$ \\
\hline$G_{32}$ & 1402 & 1394.50 & 1402 & 1393.60 & 1400 & 1392.80 & 1398 & 1387.60 & 1392 & 1381.90 \\
\hline$G_{33}$ & 1376 & 1371.00 & 1378 & 1372.00 & 1376 & 1371.80 & 1374 & 1370.70 & 1372 & 1368.10 \\
\hline$G_{35}$ & 7671 & 7654.75 & 7672 & 7660.85 & 7673 & 7663.30 & 7670 & 7661.00 & 7672 & 7650.35 \\
\hline$G_{40}$ & 2382 & 2352.35 & 2386 & 2360.85 & 2388 & 2369.70 & 2391 & 2370.20 & 2392 & 2375.50 \\
\hline$G_{41}$ & 2382 & 2354.40 & 2401 & 2364.00 & 2396 & 2369.20 & 2394 & 2375.95 & 2399 & 2376.10 \\
\hline$G_{42}$ & 2466 & 2449.60 & 2475 & 2457.75 & 2476 & 2465.95 & 2470 & 2457.55 & 2473 & 2456.70 \\
\hline$G_{55}$ & 10235 & 10211.80 & 10249 & 10229.40 & 10263 & 10231.20 & 10265 & 10233.70 & 10267 & 10238.20 \\
\hline$G_{57}$ & 3448 & 3433.00 & 3452 & 3438.80 & 3460 & 3446.20 & 3458 & 3447.30 & 3458 & 3448.90 \\
\hline$G_{62}$ & 4796 & 4775.50 & 4806 & 4790.50 & 4812 & 4792.00 & 4810 & 4795.80 & 4812 & 4791.00 \\
\hline$G_{65}$ & 5482 & 5455.70 & 5486 & 5467.70 & 5504 & 5476.50 & 5498 & 5479.90 & 5498 & 5477.50 \\
\hline$G_{66}$ & 6258 & 6230.90 & 6266 & 6245.90 & 6282 & 6259.50 & 6284 & 6250.90 & 6286 & 6258.00 \\
\hline
\end{tabular}

though improved results would be possible by further fine turning the parameters. As we see below, the adopted parameter settings are good and robust enough to allow the algorithm to attain very competitive results for the set of the tested instances compared with those reported in the literature.

\subsection{Experimental protocol}

Our MAMBP algorithm is programmed in C and compiled using GNU GCC on a PC running Windows XP with an Intel Xeon E5440 processor $(2.83 \mathrm{GHz}$ and 8G RAM).

Given the stochastic nature of MAMBP, each instance is independently solved 20 times with different random seeds. Each run is stopped when the processing time reaches its timeout limit. The timeout limit is set to be 30 minutes for graphs with $|V|<5000$ while for graphs with $|V| \geq 5000$ a time limit of 120 minutes is allowed. Note that our timeout limit condition is comparable with the stop condition reported in $[28,36]$.

Two comparisons are performed in order to assess the performance of the proposed memetic algorithm. The first comparison focuses on the max-bisection problem while the second one contrasts our (balanced) bisections with partitions obtained by several state of the art max-cut algorithms $[5,9,28,31,35,36]$. Notice that for the case of max-cut, the two subsets of a partition obtained by a max-cut algorithm are not required to be of equal cardinality. In this sense, a max-cut algorithm would have more opportunities to find partitions of larger weights. The best bisections obtained by our memetic algorithm are 
available from: http://www.info.univ-angers.fr/pub/hao/MAMBP.html

\subsection{Comparison with state-of-art max-bisection algorithm}

In this section, we present the equal sized bisections obtained by our memetic approach for the max-bisection problem. To assess the relative quality of our results, we compare MAMBP with the new Lagrangian net algorithm (NLNA) which was published very recently [38]. NLNA was run on a Lenovo PC with an Intel Core E5300 processor (2.36 GHZ CPU and 1.96 GB RAM). According to the Standard Performance Evaluation Cooperation (www.spec.org), this computer is 1.2 time slower than the computer we used for our experiments. Note that in the literature there are at least two other heuristic bisection algorithms based on deterministic annealing [7] and variable neighborhood search [25]. Unfortunately, the results reported in these references cannot be reproduced since the graphs used are not available to the public domain. As a consequence, it is impossible to compare with their results.

Table 5 presents the detailed computational results of our MAMBP algorithm in comparison with those of the reference algorithm (NLNA) reported in [38]. The first two columns in the table indicate the name of and the number of vertices of the graph. Columns 3 to 7 show MAMBP's results including the best objective value $\left(f_{\text {best }}\right)$, the success rate $($ hit $)$ for reaching $f_{\text {best }}$, the averaged objective value $\left(f_{\text {avg }}(s t d)\right)$ over the 20 runs with the standard deviation between parentheses for a hit value smaller than $100 \%$, the average iterations (Iter) and the average CPU time in seconds (time) over the 20 runs on which the $f_{\text {best }}$ value is reached. Columns 8 to 9 present NLNA's results. Given the speed ratio between our computer and the computer used for NLNA, we normalize the computing times of NLNA by dividing them with a factor of 1.2. The last column $(\Delta)$ indicates the differences in the best objective values between MAMBP and NLNA.

From Table 5, one observes that, for the 56 instances where the NLNA algorithm reports its results, our MAMBP algorithm dominates NLNA in terms of solution quality. For each of these instances, our MAMBP algorithm is able to attain a much larger objective value $\left(f_{\text {best }}\right)$ compared with NLNA. Even our average results $\left(f_{\text {avg }}\right)$ are better than the best results reported by NLNA. For the other 15 instances, no results are reported by NLNA ("-" in Table 5 indicates that a result for that particular instance was not available).

Finally, concerning the computing times, the times indicated are those that are needed for each algorithm to attain its $f_{\text {best }}$ values. To verify that our MAMBP algorithm can easily attain the best objective values of NLNA, we rerun MAMBP and use NLNA's best objective values as our stop conditions. 
Although we do not show the detailed results, this second experiment show that for the instances with less than 5000 vertices, MAMBP attains NLNA's best objective value in less than one second (against 3.17 to 58.18 seconds for NLNA). For larger graphs with at least 5000 vertices, MAMBP discovers NLNA's best objective value in no more than 10 seconds (against 260.91 to 512.62 seconds for NLNA). It is clear that MAMBP needs much less computing time than NLNA to discover solutions of comparable quality.

We can conclude that our MAMBP algorithm dominates the reference NLNA algorithm both in terms of solution quality and computing time.

\begin{tabular}{|c|c|c|c|c|c|c|c|c|c|}
\hline \multirow[t]{2}{*}{ Instance } & \multirow[t]{2}{*}{$|V|$} & \multicolumn{5}{|c|}{$M A M B P$} & \multicolumn{2}{|c|}{$N L N A[38]$} & \multirow[t]{2}{*}{$\Delta$} \\
\hline & & $f_{\text {best }}$ & $f_{a v g}(s t d)$ & hit & Iter & time & $f_{\text {best }}$ & time & \\
\hline$G_{1}$ & 800 & 11624 & 11624 & $20 / 20$ & 186225 & 2.40 & 11490 & 22.22 & 134 \\
\hline$G_{2}$ & 800 & 11617 & 11617 & $20 / 20$ & 457273 & 5.20 & 11505 & 21.95 & 112 \\
\hline$G_{3}$ & 800 & 11621 & 11621 & $20 / 20$ & 54756 & 1.32 & 11511 & 21.95 & 110 \\
\hline$G_{4}$ & 800 & 11646 & 11646 & $20 / 20$ & 183623 & 1.77 & 11554 & 22.04 & 92 \\
\hline$G_{5}$ & 800 & 11631 & 11631 & $20 / 20$ & 35178 & 0.88 & 11521 & 21.80 & 110 \\
\hline$G_{6}$ & 800 & 2177 & 2177 & $20 / 20$ & 83819 & 1.16 & 2037 & 22.08 & 140 \\
\hline$G_{7}$ & 800 & 2002 & 2002 & $20 / 20$ & 91160 & 0.82 & 1889 & 22.00 & 113 \\
\hline$G_{8}$ & 800 & 2004 & 2004 & $20 / 20$ & 312230 & 4.26 & 1873 & 21.94 & 131 \\
\hline$G_{9}$ & 800 & 2052 & 2052 & $20 / 20$ & 202926 & 1.19 & 1907 & 21.86 & 145 \\
\hline$G_{10}$ & 800 & 1998 & 1998 & $20 / 20$ & 555555 & 5.59 & 1875 & 21.96 & 123 \\
\hline$G_{11}$ & 800 & 564 & 564 & $20 / 20$ & 847410 & 12.10 & 560 & 3.18 & 4 \\
\hline$G_{12}$ & 800 & 556 & 556 & $20 / 20$ & 810531 & 11.54 & 546 & 3.17 & 10 \\
\hline$G_{13}$ & 800 & 582 & 582 & $20 / 20$ & 14481952 & 32.52 & 572 & 3.17 & 10 \\
\hline$G_{14}$ & 800 & 3062 & 3062 & $20 / 20$ & 165369788 & 799.00 & 3023 & 7.02 & 39 \\
\hline$G_{15}$ & 800 & 3050 & 3050 & $20 / 20$ & 125879080 & 692.96 & 2996 & 7.01 & 54 \\
\hline$G_{16}$ & 800 & 3052 & 3052 & $20 / 20$ & 28611328 & 82.82 & 2994 & 7.02 & 58 \\
\hline$G_{17}$ & 800 & 3047 & 3047 & $20 / 20$ & 257435761 & 778.67 & 2997 & 6.99 & 53 \\
\hline$G_{18}$ & 800 & 992 & 992 & $20 / 20$ & 3210382 & 16.36 & 909 & 7.03 & 83 \\
\hline$G_{19}$ & 800 & 905 & 905 & $20 / 20$ & 13295276 & 40.31 & 823 & 7.00 & 82 \\
\hline$G_{20}$ & 800 & 941 & 941 & $20 / 20$ & 275771 & 2.48 & 865 & 6.98 & 76 \\
\hline$G_{21}$ & 800 & 930 & 930 & $20 / 20$ & 6733404 & 34.71 & 849 & 6.98 & 81 \\
\hline$G_{22}$ & 2000 & 13359 & 13359 & $20 / 20$ & 49429658 & 303.20 & 13105 & 57.48 & 254 \\
\hline$G_{23}$ & 2000 & 13344 & 13344 & $20 / 20$ & 33121221 & 132.13 & 13120 & 57.36 & 224 \\
\hline$G_{24}$ & 2000 & 13336 & 13336 & $20 / 20$ & 25855349 & 102.75 & 13115 & 57.34 & 221 \\
\hline$G_{25}$ & 2000 & 13340 & 13340 & $20 / 20$ & 76664567 & 308.51 & 13125 & 57.41 & 215 \\
\hline$G_{26}$ & 2000 & 13328 & 13328 & $20 / 20$ & 56585007 & 366.09 & 13160 & 57.25 & 168 \\
\hline$G_{27}$ & 2000 & 3341 & 3341 & $20 / 20$ & 17155489 & 109.49 & 3109 & 57.16 & 232 \\
\hline$G_{28}$ & 2000 & 3298 & 3298 & $20 / 20$ & 34382069 & 217.84 & 3063 & 58.13 & 235 \\
\hline$G_{29}$ & 2000 & 3403 & 3403 & $20 / 20$ & 3121 & 1.36 & 3179 & 58.06 & 224 \\
\hline$G_{30}$ & 2000 & 3412 & 3412 & $20 / 20$ & 11178762 & 44.82 & 3139 & 58.18 & 273 \\
\hline$G_{31}$ & 2000 & 3309 & 3309 & $20 / 20$ & 41271756 & 263.21 & 3092 & 58.13 & 217 \\
\hline$G_{32}$ & 2000 & 1410 & 1410 & $20 / 20$ & 139405200 & 887.50 & 1382 & 16.88 & 28 \\
\hline$G_{33}$ & 2000 & 1382 & 1382 & $20 / 20$ & 132970476 & 856.80 & 1344 & 17.01 & 38 \\
\hline$G_{34}$ & 2000 & 1384 & 1384 & $20 / 20$ & 82098799 & 536.12 & 1350 & 16.88 & 34 \\
\hline$G_{35}$ & 2000 & 7686 & $7684.70(1.93)$ & $11 / 20$ & 168054486 & 1312.42 & 7548 & 39.22 & 138 \\
\hline$G_{36}$ & 2000 & 7678 & $7676.20(2.29)$ & $12 / 20$ & 158749652 & 1259.10 & 7530 & 39.08 & 148 \\
\hline$G_{37}$ & 2000 & 7689 & $7687.80(1.81)$ & $8 / 20$ & 200196553 & 1543.36 & 7541 & 39.21 & 148 \\
\hline$G_{38}$ & 2000 & 7688 & 7688 & $20 / 20$ & 132667831 & 922.66 & 7537 & 39.23 & 151 \\
\hline$G_{39}$ & 2000 & 2408 & 2408 & $20 / 20$ & 212777429 & 976.95 & 2255 & 40.11 & 153 \\
\hline$G_{40}$ & 2000 & 2400 & $2398.20(5.41)$ & $18 / 20$ & 227544366 & 1198.28 & 2189 & 40.00 & 211 \\
\hline$G_{41}$ & 2000 & 2405 & 2405 & $20 / 20$ & 111406876 & 546.57 & 2234 & 40.03 & 171 \\
\hline
\end{tabular}


Table 5 - continued from previous page

\begin{tabular}{|c|c|c|c|c|c|c|c|c|c|}
\hline \multirow[t]{2}{*}{ Instance } & \multirow[t]{2}{*}{$|V|$} & \multicolumn{5}{|c|}{$M A M B P$} & \multicolumn{2}{|c|}{$N L N A[38]$} & \multirow[t]{2}{*}{$\Delta$} \\
\hline & & $f_{\text {best }}$ & $f_{\text {avg }}(s t d)$ & hit & Iter & time $(s)$ & $f_{\text {best }}$ & time $(s)$ & \\
\hline$G_{42}$ & 2000 & 2481 & $2477.50(6.19)$ & $8 / 20$ & 325913745 & 1513.96 & 2290 & 40.11 & 191 \\
\hline$G_{43}$ & 1000 & 6659 & 6659 & $20 / 20$ & 135951 & 1.25 & 6580 & 15.34 & 79 \\
\hline$G_{44}$ & 1000 & 6650 & 6650 & $20 / 20$ & 1797956 & 1.18 & 6548 & 15.33 & 102 \\
\hline$G_{45}$ & 1000 & 6654 & 6654 & $20 / 20$ & 1089575 & 4.23 & 6513 & 15.33 & 141 \\
\hline$G_{46}$ & 1000 & 6649 & 6649 & $20 / 20$ & 2886106 & 10.48 & 6538 & 15.33 & 111 \\
\hline$G_{47}$ & 1000 & 6657 & 6657 & $20 / 20$ & 1650153 & 5.97 & 6529 & 15.34 & 128 \\
\hline$G_{48}$ & 3000 & 6000 & 6000 & $20 / 20$ & 13128 & 1.42 & - & - & - \\
\hline$G_{49}$ & 3000 & 6000 & 6000 & $20 / 20$ & 25179 & 1.28 & - & - & - \\
\hline$G_{50}$ & 3000 & 5880 & 5880 & $20 / 20$ & 1509358 & 33.89 & - & - & - \\
\hline$G_{51}$ & 1000 & 3847 & 3847 & $20 / 20$ & 90999846 & 292.60 & 3773 & 10.58 & 114 \\
\hline$G_{52}$ & 1000 & 3851 & 3851 & $20 / 20$ & 245428916 & 814.96 & 3788 & 10.61 & 63 \\
\hline$G_{53}$ & 1000 & 3850 & 3850 & $20 / 20$ & 133669193 & 516.28 & 3784 & 10.60 & 66 \\
\hline$G_{54}$ & 1000 & 3851 & 3851 & $20 / 20$ & 150196875 & 551.51 & 3789 & 10.63 & 62 \\
\hline$G_{55}$ & 5000 & 10299 & $10291.50(8.36)$ & $2 / 20$ & 454657489 & 2396.84 & - & - & - \\
\hline$G_{56}$ & 5000 & 4016 & $4007.30(9.14)$ & $3 / 20$ & 354003885 & 1886.98 & - & - & - \\
\hline$G_{57}$ & 5000 & 3488 & $3475.60(12.98)$ & $2 / 20$ & 343206720 & 4883.34 & - & - & - \\
\hline$G_{58}$ & 5000 & 19276 & $19265.10(11.28)$ & $1 / 20$ & 466659576 & 4276.67 & 18931 & 268.71 & 345 \\
\hline$G_{59}$ & 5000 & 6085 & $6070.80(15.27)$ & $1 / 20$ & 476554320 & 4446.16 & 5578 & 260.91 & 507 \\
\hline$G_{60}$ & 7000 & 14186 & $14172.90(10.15)$ & $1 / 20$ & 484216617 & 5508.45 & - & - & - \\
\hline$G_{61}$ & 7000 & 5796 & $5782.30(15.82)$ & $2 / 20$ & 515858117 & 3755.71 & - & - & - \\
\hline$G_{62}$ & 7000 & 4866 & $4848.20(16.00)$ & $1 / 20$ & 399958710 & 4652.00 & - & - & - \\
\hline$G_{63}$ & 7000 & 26754 & $26725.20(24.91)$ & $2 / 20$ & 253009370 & 5670.30 & - & - & - \\
\hline$G_{64}$ & 7000 & 8731 & $8707.60(17.21)$ & $1 / 20$ & 495005836 & 5793.56 & - & - & - \\
\hline$G_{65}$ & 8000 & 5556 & $5538.60(17.45)$ & $1 / 20$ & 389509251 & 5385.86 & 5418 & 290.72 & 132 \\
\hline$G_{66}$ & 9000 & 6352 & $6332.50(21.18)$ & $2 / 20$ & 260212500 & 6267.15 & 6194 & 391.03 & 154 \\
\hline$G_{67}$ & 10000 & 6934 & $6923.60(9.97)$ & $1 / 20$ & 374288575 & 6203.44 & 6782 & 512.62 & 152 \\
\hline$G_{70}$ & 10000 & 9580 & $9565.00(17.36)$ & $2 / 20$ & 247649652 & 7032.70 & - & - & - \\
\hline$G_{72}$ & 10000 & 6990 & $6972.20(17.34)$ & $2 / 20$ & 373117581 & 7046.03 & - & - & - \\
\hline$G_{77}$ & 14000 & 9900 & $9881.10(17.41)$ & $1 / 20$ & 251667626 & 6752.26 & - & - & - \\
\hline$G_{81}$ & 20000 & 13978 & $13961.00(15.03)$ & $1 / 20$ & 76080159 & 7023.49 & - & - & - \\
\hline
\end{tabular}

\subsection{Comparison with state-of-art dedicated max-cut algorithms}

In this section, we further assess the performance of our MAMBP approach by comparing our results for MBP (i.e., equal sized partitions) with those obtained with different max-cut algorithms (i.e., not necessarily equal sized partitions). Recall that the max-bisection problem is constrained by the equal cardinality requirement for the two parts of its partitions while the max-cut problem accepts any two-part partitions. Consequently, a solution for the MBP is always a solution for the MCP, but the reverse is not true. In this section, we show that even with this equal cardinality constraint, our max-bisection algorithm MAMBP is able to find solutions that are better than the state of the art max-cut algorithms.

For this comparison, we are mainly interested in solution quality, i.e., the largest sum of the weights of the cutting edges of a partition. Due to the differences among the programming languages, data structures, compiler options and computers, we don't focus on computing time. We just mention that 
the timeout limits we used are quite similar to those adopted by two recent reference algorithms $[28,36]$.

The comparative results are summarized in Table 6 on the $69 G$-set benchmark instances excluding the two largest graphs $G 77$ and $G 81$ for which no results are available for the reference algorithms. Columns 1 and 2 respectively give the instance name and the previous best solution value $f_{\text {max_cut }}^{*}$ from references $[1,5,9,23,28,31,35,36]$ (most of them are dedicated max-cut algorithms). Column 3 recalls the best results of our MAMBP approach extracted from Table 5. Columns 4-9 present the best results obtained by 6 reference algorithms: SS [28], TS-UBQP [23], DSA [1], VNSPR [9], CirCut [5] and GRASP-TS/PM [36]. The last three rows summarize the comparison between these algorithms and ours. The rows 'Better', 'Equal' and 'Worse' respectively denote the number of instances for which our MAMBP algorithm attains a result of better, equal and worse quality than each reference algorithm. We mark in bold those results that are the updated best known values obtained by the MAMBP approach.

From Table 6, we observe that our MAMBP algorithm is able to improve the current best known results in the literature for 31 instances while equaling the best known results for 25 other instances. This is a remarkable performance given that these best known results are the joint results of 2 or more max-cut algorithms. Indeed, when one compares our MAMBP algorithm with each of the 6 reference algorithms (SS, TS-UBQP, DSA, VNSPR, CirCut amd GRASP-TS/PM), we observe that MAMBP attains worse results for at most 10 instances while better results for at least 25 instances. These comparisons provide further evidence of the quality of the partitions achieved by our MAMBP algorithm.

On the other hand, on 13 instances, MAMBP's results are slightly inferior to the best known results. It is interesting to notice that most of these instances are of small size (with no more than 800 vertices). One possible explanation could be that for these graphs, the best max-cut solution is such that the two parts of the partition are of unequal size and thus not included in the search space of our MAMBP algorithm.

To sum, even if our MAMBP algorithm operates within a constrained search space composed solely of bisections (i.e., partitions of equal parts), its performance is remarkable for the max-cut problem when MAMBP is compared with dedicated max-cut algorithms. 
Table 6: Comparative results of our MAMBP algorithm with the results of 6 state of the art max-cut algorithms on the $G$-set benchmark instances. Notice that max-cut algorithms lead to a partition whose two sets $V_{1}$ and $V_{2}$ may be of different sizes contrary to a partition of MAMBP whose two sets are always of equal size.

\begin{tabular}{|c|c|c|c|c|c|c|c|c|}
\hline \multirow[t]{2}{*}{ Instance } & \multirow[t]{2}{*}{$f_{\text {max_cut }}^{*}$} & \multirow[t]{2}{*}{$f_{M A M B P}$} & \multicolumn{6}{|c|}{6 reference max-cut algorithms } \\
\hline & & & $\mathrm{SS}[28]$ & $\begin{array}{c}\text { TS- } \\
\text { UBQP [23] }\end{array}$ & $\mathrm{DSA}[1]$ & VNSPR[9] & CirCut $[5]$ & $\begin{array}{l}\text { GRASP- } \\
\text { TS/PM[36] }\end{array}$ \\
\hline$G_{1}$ & 11624 & 11624 & 11624 & 11624 & - & 11621 & 11624 & 11624 \\
\hline$G_{2}$ & 11620 & 11617 & 11620 & 11620 & - & 11615 & 11617 & 11620 \\
\hline$G_{3}$ & 11622 & 11621 & 11622 & 11620 & - & 11622 & 11622 & 11620 \\
\hline$G_{4}$ & 11646 & 11646 & 11646 & 11646 & - & 11600 & 11641 & 11646 \\
\hline$G_{5}$ & 11631 & 11631 & 11631 & 11631 & - & 11598 & 11627 & 11631 \\
\hline$G_{6}$ & 2178 & 2177 & 2165 & 2178 & - & 2102 & 2178 & 2178 \\
\hline$G_{7}$ & 2006 & 2002 & 1982 & 2006 & - & 1906 & 2003 & 2006 \\
\hline$G_{8}$ & 2005 & 2004 & 1986 & 2005 & - & 1908 & 2003 & 2005 \\
\hline$G_{9}$ & 2054 & 2052 & 2040 & 2054 & - & 1998 & 2048 & 2054 \\
\hline$G_{10}$ & 2000 & 1998 & 1993 & 2000 & - & 1910 & 1994 & 2000 \\
\hline$G_{11}$ & 564 & 564 & 562 & 564 & 542 & 564 & 560 & 564 \\
\hline$G_{12}$ & 556 & 556 & 552 & 556 & 540 & 556 & 552 & 556 \\
\hline$G_{13}$ & 582 & 582 & 578 & 580 & 564 & 580 & 574 & 582 \\
\hline$G_{14}$ & 3064 & 3062 & 3060 & 3061 & 2982 & 3055 & 3058 & 3063 \\
\hline$G_{15}$ & 3050 & 3050 & 3049 & 3050 & 2975 & 3043 & 3049 & 3050 \\
\hline$G_{16}$ & 3052 & 3052 & 3045 & 3052 & - & 3043 & 3045 & 3052 \\
\hline$G_{17}$ & 3047 & 3047 & 3043 & 3046 & - & 3030 & 3037 & 3047 \\
\hline$G_{18}$ & 992 & 992 & 988 & 991 & - & 916 & 978 & 992 \\
\hline$G_{19}$ & 906 & 905 & 903 & 904 & - & 838 & 888 & 906 \\
\hline$G_{20}$ & 941 & 941 & 941 & 941 & 876 & 900 & 941 & 941 \\
\hline$G_{21}$ & 931 & 930 & 930 & 930 & 855 & 902 & 931 & 931 \\
\hline$G_{22}$ & 13359 & 13359 & 13346 & 13359 & 12989 & 13295 & 13346 & 13349 \\
\hline$G_{23}$ & 13342 & 13344 & 13317 & 13342 & 13006 & 13290 & 13317 & 13332 \\
\hline$G_{24}$ & 13337 & 13336 & 13303 & 13337 & 12985 & 13276 & 13314 & 13324 \\
\hline$G_{25}$ & 13332 & 13340 & 13320 & 13332 & - & 12298 & 13326 & 13326 \\
\hline$G_{26}$ & 13328 & 13328 & 13294 & 13328 & - & 12290 & 13314 & 13313 \\
\hline$G_{27}$ & 3336 & 3341 & 3318 & 3336 & - & 3296 & 3306 & 3325 \\
\hline$G_{28}$ & 3295 & 3298 & 3285 & 3295 & - & 3220 & 3260 & 3287 \\
\hline$G_{29}$ & 3394 & 3403 & 3389 & 3391 & - & 3303 & 3376 & 3394 \\
\hline$G_{30}$ & 3403 & 3412 & 3403 & 3403 & 3080 & 3320 & 3385 & 3402 \\
\hline$G_{31}$ & 3302 & 3309 & 3288 & 3288 & 2936 & 3302 & 3285 & 3299 \\
\hline$G_{32}$ & 1410 & 1410 & 1398 & 1406 & 1338 & 1396 & 1390 & 1406 \\
\hline$G_{33}$ & 1382 & 1382 & 1362 & 1378 & 1330 & 1376 & 1360 & 1374 \\
\hline$G_{34}$ & 1384 & 1384 & 1364 & 1378 & 1334 & 1372 & 1368 & 1376 \\
\hline$G_{35}$ & 7685 & 7686 & 7668 & 7678 & - & 7635 & 7670 & 7661 \\
\hline$G_{36}$ & 7677 & 7678 & 7660 & 7670 & - & 7632 & 7660 & 7660 \\
\hline$G_{37}$ & 7689 & 7689 & 7664 & 7682 & - & 7643 & 7666 & 7670 \\
\hline$G_{38}$ & 7683 & 7688 & 7681 & 7683 & - & 7602 & 7646 & 7670 \\
\hline$G_{39}$ & 2397 & 2408 & 2393 & 2397 & - & 2303 & 2395 & 2397 \\
\hline$G_{40}$ & 2392 & 2400 & 2374 & 2390 & - & 2302 & 2387 & 2392 \\
\hline$G_{41}$ & 2400 & 2405 & 2386 & 2400 & - & 2298 & 2398 & 2398 \\
\hline$G_{42}$ & 2474 & 2481 & 2457 & 2469 & - & 2390 & 2469 & 2474 \\
\hline$G_{43}$ & 6660 & 6659 & 6656 & 6660 & - & 6659 & 6656 & 6660 \\
\hline$G_{44}$ & 6650 & 6650 & 6648 & 6639 & - & 6642 & 6643 & 6649 \\
\hline$G_{45}$ & 6654 & 6654 & 6642 & 6652 & - & 6646 & 6652 & 6654 \\
\hline$G_{46}$ & 6649 & 6649 & 6634 & 6649 & - & 6630 & 6645 & 6649 \\
\hline$G_{47}$ & 6656 & 6657 & 6649 & 6656 & - & 6640 & 6656 & 6656 \\
\hline$G_{48}$ & 6000 & 6000 & 6000 & 6000 & 6000 & 6000 & 6000 & 6000 \\
\hline$G_{49}$ & 6000 & 6000 & 6000 & 6000 & 6000 & 6000 & 6000 & 6000 \\
\hline$G_{50}$ & 5880 & 5880 & 5880 & 5880 & 5880 & 5880 & 5880 & 5880 \\
\hline$G_{51}$ & 3847 & 3847 & 3846 & 3847 & - & 3808 & 3837 & 3847 \\
\hline$G_{52}$ & 3850 & 3851 & 3849 & 3849 & - & 3816 & 3833 & 3850 \\
\hline$G_{53}$ & 3848 & 3850 & 3846 & 3848 & - & 3802 & 3842 & 3848 \\
\hline
\end{tabular}


Table 6 - continued from previous page

\begin{tabular}{|c|c|c|c|c|c|c|c|c|}
\hline \multirow[t]{2}{*}{ Instance } & \multirow[t]{2}{*}{$f_{\text {max_cut }}^{*}$} & \multirow[t]{2}{*}{$f_{M A M B P}$} & \multicolumn{6}{|c|}{6 reference max cut algorithms } \\
\hline & & & $\mathrm{SS}[28]$ & $\begin{array}{c}\text { TS- } \\
\text { UBQP[23] }\end{array}$ & $\overline{\mathrm{DSA}}[1]$ & VNSPR[9] & CirCut $[5]$ & $\begin{array}{l}\text { GRASP- } \\
\text { TS/PM[36] }\end{array}$ \\
\hline$G_{54}$ & 3851 & 3851 & 3846 & 3851 & - & 3820 & 3842 & 3850 \\
\hline$G_{55}$ & 10236 & 10299 & - & 10236 & 9960 & - & - & - \\
\hline$G_{56}$ & 3934 & 4016 & - & 3934 & 3649 & - & - & - \\
\hline$G_{57}$ & 3460 & 3488 & - & 3460 & 3220 & - & - & - \\
\hline$G_{58}$ & 19248 & 19276 & - & 19248 & - & - & - & - \\
\hline$G_{59}$ & 6019 & 6085 & - & 6019 & - & - & - & - \\
\hline$G_{60}$ & 14057 & 14186 & - & 14057 & 13658 & - & - & - \\
\hline$G_{61}$ & 5680 & 5796 & - & 5680 & 5273 & - & - & - \\
\hline$G_{62}$ & 4822 & 4866 & - & 4822 & 4612 & - & - & - \\
\hline$G_{63}$ & 26963 & 26754 & - & 26963 & 8059 & - & - & - \\
\hline$G_{64}$ & 8610 & 8731 & - & 8610 & 7861 & - & - & - \\
\hline$G_{65}$ & 5518 & 5556 & - & 5518 & - & - & - & - \\
\hline$G_{66}$ & 6304 & 6352 & - & 6304 & - & - & - & - \\
\hline$G_{67}$ & 6894 & 6934 & - & 6894 & - & - & - & - \\
\hline$G_{70}$ & 9499 & 9580 & - & 9458 & 9499 & - & - & - \\
\hline$G_{72}$ & 6922 & 6990 & - & 6922 & 6644 & - & - & - \\
\hline Better & & & 44 & 42 & 25 & 47 & 44 & 27 \\
\hline Equal & & & 8 & 17 & 3 & 6 & 6 & 18 \\
\hline Worse & & & 2 & 10 & 0 & 1 & 4 & 9 \\
\hline
\end{tabular}

\section{Analysis of MAMBP}

\subsection{Influence of crossover, pool replacement strategy and perturbation mech- anism}

In this section, we investigate the influence of some important ingredients of the proposed memetic algorithm: crossover (Section 2.5), population updating strategy (Section 2.6) and perturbation mechanism (Section 2.3.3). These studies are based on the same selection of the 11 hard problem instances used for setting the parameters (Section 3.2).

\subsubsection{Influence of crossover: comparing MAMBP with TS}

MAMBP uses TS as one of its main optimization procedures. It is therefore interesting to know if MAMBP, by integrating a crossover operator, is able to improve on the results of the TS algorithm alone. To verify this, we carry out additional experiments on the selection of the $11 G$-set graphs and show a comparison between MAMBP and TS. For this experiment, we use the TS algorithm described in Algorithm 2 (see Section 2.3) with the same parameter settings given in Table 1. To make the comparison as fair as possible, we run TS with the same timeout limits as for MAMBP: 30 minutes for graphs with $|V|<5000$ and 120 minutes if $|V| \geq 5000$ (see Section 3.3). In order not to penalize the tabu search algorithm which usually stops after $l r$ iterations 
Table 7

Comparative results of MAMBP and TS on $11 G$-set instances for the max-bisection problem

\begin{tabular}{|c|c|c|c|c|c|c|c|c|c|}
\hline \multirow[t]{2}{*}{ Instance } & \multirow[t]{2}{*}{$|V|$} & \multicolumn{4}{|c|}{ MAMBP } & \multicolumn{4}{|c|}{$\mathrm{TS}$} \\
\hline & & $f_{\text {best }}$ & $f_{\text {avg }}$ & hit & time $(s)$ & $f_{\text {best }}$ & $f_{\text {avg }}$ & hit & time $(s)$ \\
\hline$G_{32}$ & 2000 & 1410 & 1410 & $20 / 20$ & 887.50 & 1402 & 1400.80 & $4 / 20$ & 1519.17 \\
\hline$G_{33}$ & 2000 & 1382 & 1382 & $20 / 20$ & 856.80 & 1380 & 1378.60 & $6 / 20$ & 802.67 \\
\hline$G_{35}$ & 2000 & 7686 & 7684.70 & $11 / 20$ & 1312.42 & 7683 & 7680.35 & $1 / 20$ & 1521.15 \\
\hline$G_{40}$ & 2000 & 2400 & 2398.20 & $18 / 20$ & 1198.28 & 2400 & 2397.80 & $2 / 20$ & 1285.30 \\
\hline$G_{41}$ & 2000 & 2405 & 2405 & $20 / 20$ & 546.57 & 2405 & 2403.40 & $5 / 20$ & 982.45 \\
\hline$G_{42}$ & 2000 & 2481 & 2479.50 & $8 / 20$ & 1513.96 & 2481 & 2477.50 & $2 / 20$ & 807.34 \\
\hline$G_{55}$ & 5000 & 10299 & 10291.50 & $2 / 20$ & 2396.84 & 10291 & 10275.70 & $1 / 20$ & 1971.73 \\
\hline$G_{57}$ & 5000 & 3488 & 3475.60 & $2 / 20$ & 4883.34 & 3472 & 3466.20 & $1 / 20$ & 2833.71 \\
\hline$G_{62}$ & 7000 & 4866 & 4848.20 & $1 / 20$ & 4652.00 & 4830 & 4827.20 & $3 / 20$ & 5089.40 \\
\hline$G_{65}$ & 8000 & 5556 & 5538.60 & $1 / 20$ & 5385.86 & 5516 & 5511.00 & $2 / 20$ & 4408.32 \\
\hline$G_{66}$ & 9000 & 6352 & 6332.50 & $2 / 20$ & 6267.15 & 6298 & 6293.20 & $2 / 20$ & 5959.70 \\
\hline
\end{tabular}

$\left(l r=10^{6}\right.$ in our case as indicated in Table 1$)$, TS is restarted after $l r=10^{6}$ iterations whenever the timeout limit is not reached. Like for MAMBP, we run the tabu search algorithm 20 times on each of the 11 selected $G$-set graphs.

Table 7 summarizes the computational results of the TS algorithm in comparison with those of MAMBP taken from Table 5 . The following information for each instance is shown: the best objective value $\left(f_{\text {best }}\right)$, the averaged objective value over the 20 runs $\left(f_{\text {avg }}\right)$, the success rate $($ hit $)$ for reaching its $f_{\text {best }}$ value and the average CPU time (time) over the runs on which the $f_{\text {best }}$ value is reached. From Table 7, we notice that for all the 11 selected instances, MAMBP performs far better than TS. Especially for each of the instances with at least 5000 vertices, MAMBP is able to reach a larger objective value $f_{\text {best }}$ than TS. In addition, improvements are more pronounced for larger-size graphs. These comparative results provide clear evidence that the recombination operator plays an important role in the overall performance of our MAMBP algorithm and is more efficient when it comes to handling large graphs.

\subsubsection{Influence of population updating strategy}

In order to evaluate the distance-and-quality based population updating strategy (denoted by DisQual), we compare it with a popular updating strategy widely used in the literature (denoted by PoolWorst), which uses the new offspring solution to replace the worst solution (in terms of the fitness) of the population.

We have experimented both strategies within our memetic algorithm. As an illustration, Fig. 4 shows the detailed comparison on a large and difficult graph $\left(G_{55}\right)$. Similar results have been observed on other instances. The stopping 
criterion is the number of generations (i.e., the number of applications of the recombination operation) which is set to 300 .

We keep other ingredients unchanged in the MAMBP algorithm and observe two evolution profiles of each population updating strategy: the best objective value (over 20 runs) vs. the number of generations (Fig. 4, left) and the population diversity vs. the number of generations (Fig. 4, right). The diversity of the population is defined as the average distance between each pair of solutions in the population (see Section 2.6) [12,27,32].
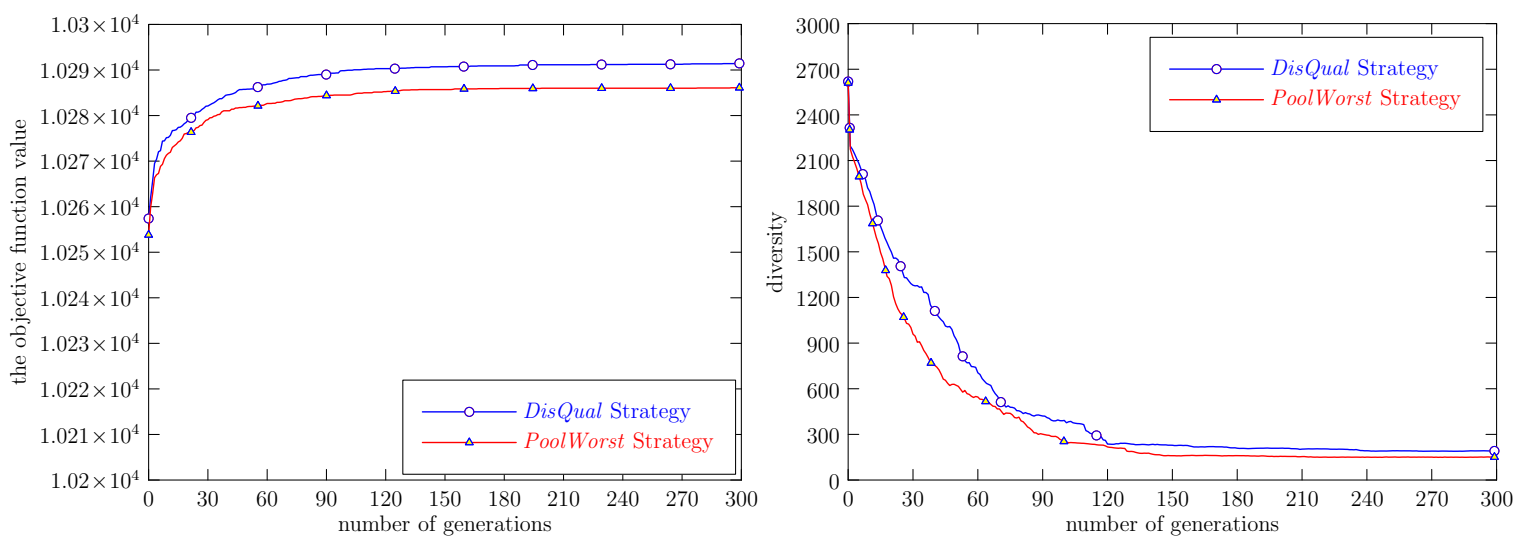

Fig. 4. Comparison between two population updating strategies

Fig. 4 (left) shows that the population converges more quickly toward high quality solutions with the distance-and-quality based population updating strategy than with the classical PoolWorst strategy. In addition, the population diversity is better preserved during the evolution process with DisQual than with PoolWorst, which is directly correlated to the evolution of the solution quality. We can conclude that the distance-and-quality updating strategy introduced in our approach allows the algorithm to better explore the search space and prevents the population from stagnating in poor local optima.

\subsection{Influence of the perturbation mechanism}

As indicated in Section 2.3.3, our tabu search procedure employs a perturbation mechanism which tries to bring controlled diversifications into the search. In order to be sure this perturbation mechanism makes a meaningful contribution, we report in Table 8 a comparison between the two versions of our tabu search algorithm with and without the perturbation mechanism (denoted by TS+PM and TS-PM respectively). We run both algorithms 20 times on the set of the 11 selected instances, each run being limited to $l r=10^{6}$ iterations. Table 8 reports the results of this experiment. For each instance, the following information is provided: the best objective value $\left(f_{\text {best }}\right)$, the averaged objective value over the 20 executions $\left(f_{\text {avg }}\right)$, and the number of executions (hit) 
Table 8

The influence of the perturbation mechanism on $11 G$-set bi-section instances

\begin{tabular}{|c|c|c|c|c|c|c|c|}
\hline \multirow[t]{2}{*}{ Instance } & \multirow[t]{2}{*}{$|V|$} & \multicolumn{3}{|c|}{ TS-PM } & \multicolumn{3}{|c|}{$\mathrm{TS}+\mathrm{PM}$} \\
\hline & & $f_{\text {best }}$ & $f_{\text {avg }}$ & hit & $f_{\text {best }}$ & $f_{\text {avg }}$ & hit \\
\hline$G_{32}$ & 2000 & 1398 & 1382.00 & $1 / 20$ & 1400 & 1392.80 & $2 / 20$ \\
\hline$G_{33}$ & 2000 & 1372 & 1355.20 & $1 / 20$ & 1376 & 1371.80 & $4 / 20$ \\
\hline$G_{35}$ & 2000 & 7665 & 7650.25 & $1 / 20$ & 7673 & 7663.30 & $1 / 20$ \\
\hline$G_{40}$ & 2000 & 2366 & 2343.85 & $2 / 20$ & 2388 & 2369.70 & $1 / 20$ \\
\hline$G_{41}$ & 2000 & 2379 & 2346.85 & $2 / 20$ & 2396 & 2369.20 & $2 / 20$ \\
\hline$G_{42}$ & 2000 & 2459 & 2430.40 & $1 / 20$ & 2476 & 2465.95 & $1 / 20$ \\
\hline$G_{55}$ & 5000 & 10004 & 9923.05 & $1 / 20$ & 10263 & 10231.20 & $1 / 20$ \\
\hline$G_{57}$ & 5000 & 3420 & 3399.60 & $1 / 20$ & 3460 & 3446.20 & $1 / 20$ \\
\hline$G_{62}$ & 7000 & 4778 & 4755.20 & $1 / 20$ & 4812 & 4792.00 & $1 / 20$ \\
\hline$G_{65}$ & 8000 & 5450 & 5423.80 & $1 / 20$ & 5504 & 5476.50 & $2 / 20$ \\
\hline$G_{66}$ & 9000 & 6226 & 6205.60 & $1 / 20$ & 6282 & 6259.50 & $1 / 20$ \\
\hline
\end{tabular}

reaching its $f_{\text {best }}$ value. Table 8 discloses that in most cases, our TS algorithm performs better with the perturbation mechanism (Column "TS+PM") than without it (Column "TS-PM"), confirming the usefulness of the perturbation mechanism within the TS algorithm.

\subsection{Landscape analysis and motivation for the proposed crossover operator}

In the last section, we demonstrated the importance of the proposed crossover operator to the overall performance of the memetic algorithm by comparing the performance of the proposed memetic algorithm with its underlying tabu search algorithm. In this section, we try to explain why our grouping crossover operator performs well and provide motivations for the proposed crossover operator. For this purpose, we analyze structural similarity between local optima of different quality in terms of the size of the commonly shared vertex groupings. Additionally, we investigate the correlation between quality (fitness) of local optima and their distances (according to Definition 1, Section 2.6) to the optimum by utilizing fitness landscape analysis techniques, which have been shown to be useful for understanding the behavior of an optimization algorithm.

To analyze structural similarity between local optima, we use the same set of the 11 selected $G$-set graphs as before. For each instance, we produce 8000 local optima of different quality using our memetic algorithm as well as its underlying tabu search with different time limits. Among these 8000 local optima, we select the top 10\% (800) with the largest objective function values and call them 'high-quality solutions'. Moreover, since the global optimal solutions for the selected instances are unknown, we use instead the best solutions found by our memetic algorithm as our supposed global optima. 
Table 9

\begin{tabular}{ccccccc} 
Analysis of structural similarity between high-quality solutions for 11 & \multicolumn{3}{c}{ hard max-bisection instances } \\
\cline { 2 - 5 } Instance & $n_{\text {distinct }}$ & $\rho_{f d c}$ & $S_{h q}$ & $S_{l o}$ & $D_{h q}$ & $D_{l o}$ \\
\hline$G_{32}$ & 200 & -0.71 & 0.64 & 0.55 & 34.10 & 64.70 \\
$G_{33}$ & 79 & -0.69 & 0.63 & 0.57 & 49.60 & 67.70 \\
$G_{35}$ & 167 & -0.69 & 0.69 & 0.57 & 32.80 & 66.50 \\
$G_{40}$ & 177 & -0.31 & 0.73 & 0.59 & 44.60 & 81.40 \\
$G_{41}$ & 613 & -0.80 & 0.93 & 0.63 & 11.40 & 67.40 \\
$G_{42}$ & 152 & -0.22 & 0.70 & 0.59 & 60.70 & 82.50 \\
$G_{55}$ & 5 & -0.19 & 0.66 & 0.56 & 63.40 & 84.00 \\
$G_{57}$ & 30 & -0.61 & 0.60 & 0.55 & 53.20 & 67.60 \\
$G_{62}$ & 26 & -0.62 & 0.59 & 0.54 & 61.00 & 75.10 \\
$G_{65}$ & 37 & -0.63 & 0.58 & 0.54 & 60.70 & 75.40 \\
$G_{66}$ & 44 & -0.62 & 0.58 & 0.53 & 60.80 & 76.90 \\
\hline
\end{tabular}

Table 9 contains the data related to the similarity between our 8000 local optima. Column $n_{\text {distinct }}$ indicates the total number of distinct global optima found for each instance. Columns $S_{h q}$ and $S_{l o}$ report respectively the average degree of similarity between the 800 high-quality solutions and the average degree of similarity between all the 8000 sampled local optima, the degree of similarity between two solutions $I^{a}$ and $I^{b}$ is expressed as $\frac{s\left(I^{a}, I^{b}\right)}{|V|}$ where $s\left(I^{a}, I^{b}\right)$ is a similarity function as described in Section 2.6. Columns $D_{h q}$ and $D_{l o}$ present respectively the average distance of high-quality solutions to their nearest global optimum and the average distance of all local optima to their nearest global optimum, expressed as a percentage of $\frac{|V|}{2}$ given that $\frac{|V|}{2}$ is the maximum distance between any two solutions.

From Table 9, we observe that in most cases, the degree of similarity between high-quality solutions is generally very large, which indicates high quality solutions share many groupings of vertices. We also notice that for all the cases, the degree of similarity between high-quality solutions is larger than that between other local optima. In addition, the values reported in ' $D_{h q}$ ' are generally smaller than the ones in ' $D_{l o}$ ', which indicates that the structure of the set of high quality solutions is very similar to the structure of the supposed global optimum. This suggests that if a significant number of vertices is grouped together throughout each of the high quality solutions, there is a strong chance that they are also grouped together in the global optimum. This observation constitutes a motivation and justification for our crossover which tries to preserve the largest common vertex groupings with respect to the two selected parent solutions.

In order to give further evidence that the $G$-set instances are well suited for our memetic algorithm, we carry out a landscape analysis and employ the fitness distance correlation (FDC) [19] to study the difficulty of the $G$-set instances for our memetic algorithm. FDC estimates how closely the fitness and distance to 

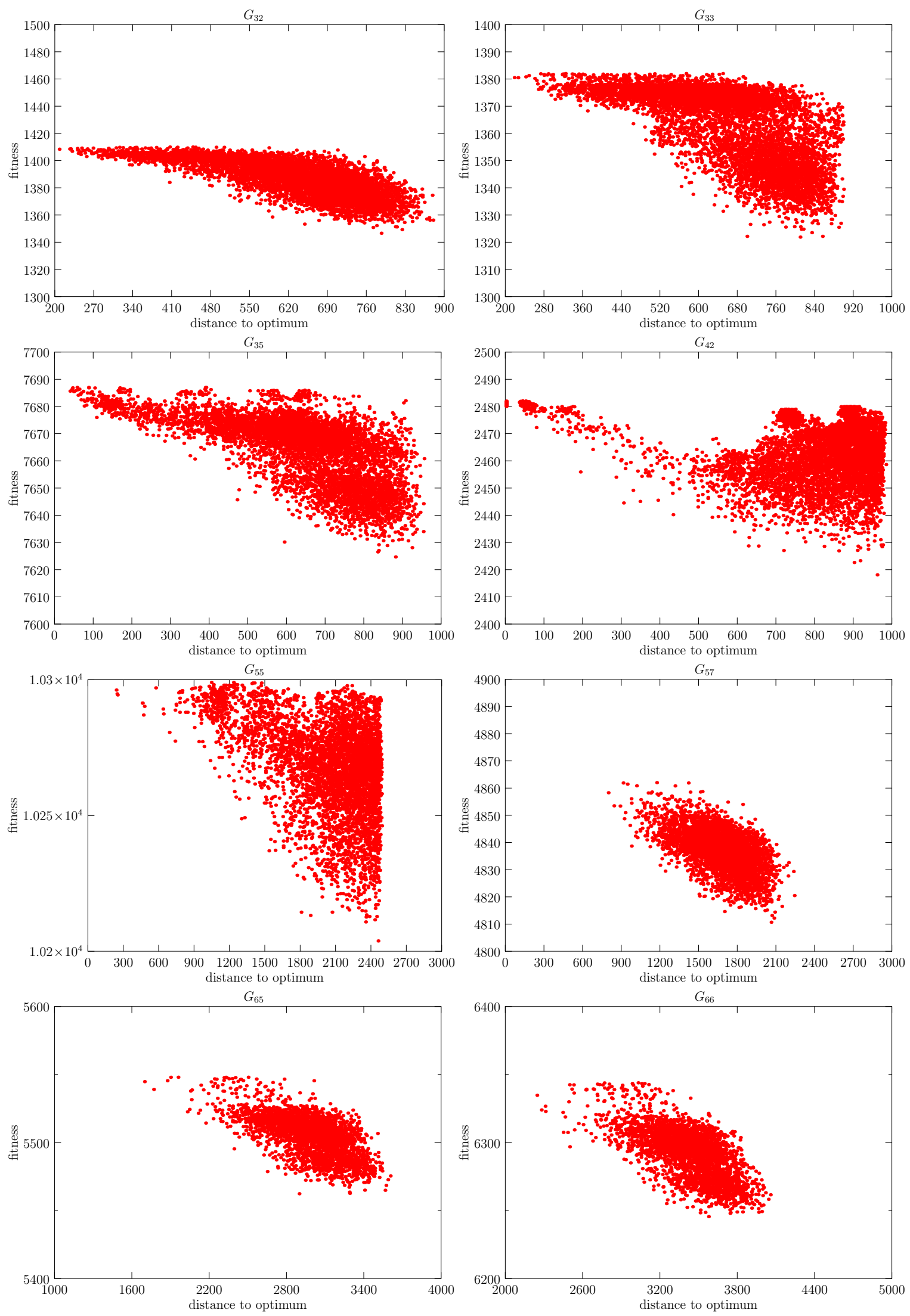

Fig. 5. Fitness-distance correlation plots with respect to the solution fitness and distance to the optimum for 8 graphs G32, G33, G35, G42, G55, G57, G65 and G66. 
the nearest global optimum are related. If fitness improves when the distance to the optimum becomes smaller, then the search space is expected to be easy for the search algorithm, since there is a "path" to the optimum via solutions with increasing fitness. A value of the fitness distance correlation coefficient $\rho_{f d c}=-1$ for a maximization problem indicates that fitness and distance to the optimum are perfectly related and that search promises to be easy. A value of $\rho_{f d c}=1$ means that the fitness function is completely misleading. FDC can also be visualized with the FD plot, where the same data used for estimating $\rho_{f d c}$ is displayed graphically. For this study, we use the same solutions as above and the best solution as our supposed global optima. FD plots of 8 of the 11 instances are given in Fig. 5.

From these plots, one observes that G42 and G55 have the weakest FD correlations with a value of $\rho_{f d c}$ equaling -0.261 and -0.193 respectively (Table 9 ). It is interesting to relate these values to the results of the memetic algorithm shown in Table 5. Indeed, for these graphs, MAMBP reaches its best solutions with a relatively low probability of $8 / 20$ and $2 / 20$ respectively. On the contrary, for the two graphs where the FC correlations are the highest (G42 and G41 with $\rho_{f d c}=-0.706$ and -0.799 ), MAMBP attains its best solutions easily with a $100 \%$ success rate. However, for the remaining graphs, the relation between the FDC and MAMBP's performance is more difficult to establish. This shows that a simple measure like FDC cannot be used to fully characterize the search difficulty of a problem instance.

\section{Conclusion}

In this paper, we have presented for the first time a memetic algorithm for the max-bisection problem (MAMBP). The proposed algorithm relies on a diversification-guided grouping crossover operator, a tabu search optimization procedure and a quality-and-diversity based population updating strategy.

The computational results obtained on the set of 71 well-known $G$-set graphs demonstrate that the proposed algorithm outperforms a recently published Lagrangian net algorithm for MBP by delivering improved solutions for each graph. Moreover, when considering the max-bisection problem as the "balanced" max-cut problem, the MAMBP algorithm remain highly competitive even compared to 6 state of the art max-cut algorithms. For 31 instances, MAMBP improves on the previous best known results reported in the literature.

Furthermore, we have carried out experiments to show the importance of the proposed crossover operator to the overall performance of the memetic algorithm. We have also shown an analysis of the similarity between high quality 
solutions and of the search landscapes to get insights into the search space and provide motivations for the proposed grouping crossover operator. We have shown experimentally the usefulness of other important ingredients like the dynamic tabu tenure management, the perturbation mechanism and the pool updating strategy.

\section{Acknowledgment}

We are grateful to the anonymous referees for valuable suggestions and comments which have helped us to improve the paper. The work is partially supported by the RaDaPop (2009-2013) and LigeRo projects (2009-2013) from Pays de la Loire Region, France.

\section{References}

[1] J.E. Beasley, Heuristic algorithms for the unconstrained binary quadratic programming problem. Working Paper, The Management School, Imperial College, London, England, 1998.

[2] T. Bäck, D. B. Fogel, Z. Michalewicz (Eds), Handbook of evolutionary computation. Springer, 1997.

[3] U. Benlic, J.K. Hao, A multilevel memetic approach for improving graph kpartitions. IEEE Transactions on Evolutionary Computation, 15(5):624-472, 2011.

[4] U. Benlic, J.K. Hao, Hybrid metaheuristics for the graph partitioning problem. In EG Talbi (Ed.) Hybrid Metaheuristics. Studies in Computational Intelligence, Chapter 9, 2012.

[5] S. Burer, R.D.C. Monteiro, Y. Zhang, Rank-two relaxation heuristics for maxcut and other binary quadratic programs. SIAM Journal on Optimization, 12(2):503-521, 2002.

[6] K.C. Chang, DH-C. Du, Efficient algorithms for layer assignment problems. IEEE Transactions on Computer-Aided Design of Integrated Circuits and Systems, 6(1):67-78, 1987.

[7] C. Dang, L. He, I. Hui, A deterministic annealing algorithm for approximating a solution of the max-bisection problem, Neural Networks, 15(3):441-458, 2002.

[8] E. Falkenauer, Genetic algorithms and grouping problems. New York: Wiley, 1998. 
[9] P. Festa, P.M. Pardalos, M.G.C. Resende, C.C. Ribeiro, Randomized heuristics for the max-cut problem. Optimization Methods and Software, 17(6):1033-1058, 2002 .

[10] A. Frieze, M. Jerrum. Improved approximation algorithm for max k-cut and max-bisection, Algorithmica, 18(1):67-81, 1997.

[11] P. Galinier, Z. Boujbel, M.C. Fernandes, An efficient memetic algorithm for the graph partitioning problem, Annals of Operations Research, 191(1):1-22, 2011.

[12] P. Galinier, J.K. Hao, Hybrid evolutionary algorithms for graph coloring, Journal of Combinatorial Optimization, 3(4):379-397, 1999.

[13] M. Garey, D. Johnson, Computers and Intractability. Freeman. N.Y., 1979.

[14] F. Glover, M. Laguna, Tabu Search, Kluwer Academic, Boston, 1997.

[15] M.X. Goemans, D.P. Williamson, Improved approximation algorithms for maximum cut and satisfiability problems using semidefinite programming, Journal of the Association for Computing Machinery, 42(6):1115-1145, 1995.

[16] E. Halperin, U. Zwick, A unified framework for obtaining improved approximation algorithms for maximum graph bisection problem, Random Struct. Algorithms, 20(3):382-402, 2002.

[17] J.K. Hao, Memetic algorithms in discrete optimization. In F. Neri, C. Cotta, P. Moscato (Eds.) Handbook of Memetic Algorithms. Studies in Computational Intelligence 379, Chapter 6, pages 73-94, 2012.

[18] B. Hendrickson, R. Leland, The Chaco User's Guide Version 2.0. Technical Report, Sandia National Laboratories, 1995.

[19] T. Jones, S. Forrest, Fitness distance correlation as a measure of problem difficulty for genetic algorithms. Proceedings of the 6 th International Conference on Genetic Algorithms, Morgan Kaufmann, 184-192, 1995.

[20] S. Karish, F. Rendl, J. Clausen, Solving graph bisection problems with semidefinite programming, SIAM Journal on Computing 12(3):177-191, 2000.

[21] R.M. Karp, Reducibility among combinatorial problems, in R. E. Miller, J. W. Thacher (Ed.), Complexity of Computer Computation, Plenum Press, pages 85-103, 1972.

[22] G. Karypis, V. Kumar, Multilevel k-way partitioning scheme for irregular graphs. Journal of Parallel and Distributed Computing, 48(1): 96-129, 1998.

[23] G. A. Kochenberger, J.K. Hao, Z. Lü, H. Wang, F. Glover, Solving large scale max cut problems via tabu search. Accepted to Journal of Heuristics to appear in 2012. DOI : 10.1007/s10732-011-9189-8

[24] K. Krishnan, J. Mitchell, A semidefinite programming based polyhedral cut and price approach for the Max-Cut problem. Computational Optimization and Applications, 33(1): 51-71, 2006. 
[25] A. Ling, C. Xu, L. Tang, A modified VNS metaheuristic for max-bisection problems, Journal of Computational and Applied Mathematics, 220(1-2):413$421,2008$.

[26] Z. Lü, F. Glover, J.K. Hao, A hybrid metaheuristic approach to solving the UBQP problem. European Journal of Operational Research, 207(3):1254-1262, 2010 .

[27] Z. Lü, J.K. Hao, A memetic algorithm for graph coloring. European Journal of Operational Research, 203(1):241-250, 2010.

[28] R. Marti, A. Duarte, M. Laguna, Advanced scatter search for the max-cut problem. INFORMS Journal on Computing, 21(1):26-38, 2009.

[29] P. Moscato, C. Cotta, A Gentle Introduction to Memetic Algorithms. In F. Glover and G. A. Kochenberger (Eds.), Handbook of Metaheuristics. Kluwer, Norwell, Massachusetts, USA, 2003.

[30] F. Neri, C. Cotta, P. Moscato (Eds.) Handbook of Memetic Algorithms. Studies in Computational Intelligence 379, Springer, 2011.

[31] G. Palubeckis, Application of multistart tabu search to the MaxCut problem. Information Technology and Control, 2(31):29-35, 2004.

[32] D.C. Porumbel, J.K. Hao, P. Kuntz, An evolutionary approach with diversity guarantee and well-informed grouping recombination for graph coloring. Computers and Operations Research, 37(10):1822-1832, 2010.

[33] D.C. Porumbel, J.K. Hao, P. Kuntz, An efficient algorithm for computing the distance between close partitions. Discrete Applied Mathematics, 159(1): 53-59, 2011.

[34] F. Rendl, G. Rinaldi, A. Wiegele, Solving Max-Cut to optimality by intersecting semidefinite and polyhedral relaxations, Mathematical Programming 121(2): 307-335 2008.

[35] V.P. Shylo, O.V. Shylo, Solving the maxcut problem by the global equilibrium search. Cybernetics and Systems Analysis, 46(5):744-754, 2010.

[36] Y. Wang, Z. Lü, F. Glover, J.K. Hao, Probabilistic GRASP-tabu search algorithms for the UBQP problem. Accepted to Computers and Operations Research DOI: 10.1016/j.cor.2011.12.006

[37] C. Walshaw, M. Cross, Mesh partitioning: A multilevel balancing and refinement algorithm. SIAM Journal on Scientific Computing, 22(1): 63-80, 2000 .

[38] F. Xu, X. Ma, B. Chen, A new Lagrangian net algorithm for solving maxbisection problems, Journal of Computational and Applied Mathematics, 235(13):3718-3723, 2011.

[39] Y. Ye, A 0.699-approximation algorithm for max-bisection, Mathematical Programming, 90(1):101-111, 2001. 